ESAIM: COCV 19 (2013) 43-62

DOI: $10.1051 / \mathrm{cocv} / 2011197$
ESAIM: Control, Optimisation and Calculus of Variations

www.esaim-cocv.org

\title{
EXISTENCE OF OPTIMAL NONANTICIPATING CONTROLS IN PIECEWISE DETERMINISTIC CONTROL PROBLEMS
}

\author{
AtLe Seierstad ${ }^{1}$
}

\begin{abstract}
Optimal nonanticipating controls are shown to exist in nonautonomous piecewise deterministic control problems with hard terminal restrictions. The assumptions needed are completely analogous to those needed to obtain optimal controls in deterministic control problems. The proof is based on well-known results on existence of deterministic optimal controls.
\end{abstract}

Mathematics Subject Classification. 93E20.

Received August 24, 2010. Revised January 28, 2011.

Published online 18 January 2012.

\section{INTRODUCTION}

In this paper, optimal nonanticipating controls are shown to exist in nonautonomous piecewise deterministic control problems. The assumptions needed for obtaining existence are completely analogous to those needed to obtain optimal controls in the simplest cases in deterministic control problems, namely a common bound on admissible solutions, compactness of the control region and, essentially, convexity of the velocity set. The proof mainly involves standard arguments and include the use of well-known results on existence of deterministic optimal controls.

In a certain sense, the nonautonomy in the problem means that existence arguments, carried out once in the stationary case, now have to be repeated an infinite number of time. The hard restrictions means that the optimal value functions used in the proof take on the value $-\infty$, in case the restrictions cannot be met.

Normally, one can transform a nonautonomous problem to a stationary one, but in the present context, it does not seem to give any great advantages, and the proof would be less transparent.

Existence theorems for nonrelaxed controls involving convexity condition are given in Dempster and Ye [7], and for another type of condition in Forwick et al. [8], (for relaxed controls, see e.g. Davis [3]). In contrast to the works mentioned, the present paper treats nonautonomous problems and hard terminal restrictions (restrictions required to hold a.s.), and obtains existence of optimal controls dependent on previous jump times, so-called nonanticipating controls ${ }^{2}$. The references include also works treating necessary and/or sufficient conditions (including verification principles).

Keywords and phrases. Piecewise deterministic problems, optimal controls, existence.

1 University of Oslo, Department of Economics, Box 1095 Blindern, 0317 Oslo, Norway. atle.seierstad@econ.uio.no

${ }^{2}$ Seierstad [10] presents simple examples, to which the existence results below apply, that are solved by means of necessary conditions in the form of a maximum principle. General necessary conditions for hard end constrained problems are proved in Seierstad [11]. 
First, systems where there are no jumps in the state variable are treated (there are then sudden changes in the differential equation).

\section{Sudden stochastic Changes in the Differential Equation, Continuous SOLUTIONS}

Consider the following control problem

$$
\max _{u(. . .)} E\left[\int_{0}^{T} f_{0}\left(t, x^{u(. . .)}(t, \tau), u(t, \tau), \tau\right) \mathrm{d} t+h^{*}(x(T, \tau))\right]
$$

subject to

$$
\dot{x}=f(t, x, u(t, \tau), \tau), t \in J:=[0, T], x(0)=x_{0} \in \mathbb{R}^{n}, u(t, \tau) \in U \subset \mathbb{R}^{r},
$$

and, a.s.,

$$
\begin{gathered}
x^{i}(T, \tau)=\bar{x}^{i}, \quad i=1, \ldots, n_{1}, \\
x^{i}(T, \tau) \geq \bar{x}^{i}, \quad i=n_{1}+1, \ldots, n_{2} \leq n .
\end{gathered}
$$

Here $f_{0}: J \times \mathbb{R}^{n} \times U \times \Omega \rightarrow \mathbb{R},(\Omega$ defined in a moment $), h^{*}: \mathbb{R}^{n} \rightarrow \mathbb{R}$, and $f: J \times \mathbb{R}^{n} \times U \times \Omega \rightarrow \mathbb{R}^{n}$, are fixed functions, moreover, the control region $U$, the initial point $x_{0}$, and the terminal time $T$ are also fixed, whereas the control functions $u(t, \tau)$ are subject to choice. Certain stochastic time-points $\tau_{i}, i=1,2, \ldots$, $\tau_{i}<\tau_{i+1}$, influence both the right hand side of the differential equation as well as the integrand in the criterion, as $\tau=\left(\tau_{0}, \tau_{1}, \tau_{2}, \ldots\right) \in \Omega=\left\{\left(\tau_{0}, \tau_{1}, \tau_{2}, \ldots\right): \tau_{i} \in[0, \infty)\right\}, \tau_{0}=0, \tau_{i}<\tau_{i+1}$. Thus in this type of systems, the right hand side of the differential equation (as well as the integrand in the criterion) exhibits sudden changes at stochastic points in time $\tau_{i}$. In concrete (economic) situations, these changes may be the result of earthquakes, inventions, sudden currency devaluations etc. Given $u(.,$.$) and \tau$, the differential equation is an ordinary deterministic equation with continuous solution $t \rightarrow x^{u(., .)}(t, \tau)$. (More details are given below). The solution depends of course on $\tau$, (the stochastic variable), and what we obtain is pathwise solutions. The present type of systems might be termed continuous, piecewise deterministic. The points $\tau_{i}$ are random variables taking values in $[0, \infty)$, with probability properties as follows: conditional probability densities $\mu\left(\tau_{j+1} \mid \tau_{0}, \ldots, \tau_{j}\right)$ are given, (for $j=0$, the density is simply $\mu\left(\tau_{1}\right)$, sometimes written $\mu\left(\tau_{1} \mid \tau_{0}\right), \tau_{0}=0$ ). The conditional density $\mu\left(\tau_{j+1} \mid \tau_{0}, \ldots, \tau_{j}\right)$ is assumed to be integrable with respect to $\tau_{j+1}$, with integral 1 . (We use the following conventions: Measurable $=$ Lebesgue measurable, meas $(A)=$ Lebesgue measure of $A$, integrable $=$ Lebesgue integrable). We assume $\mu\left(\tau_{j+1} \mid \tau_{0}, \ldots, \tau_{j}\right)=0$ if $\tau_{j+1}<\max _{1 \leq i \leq j} \tau_{i}$, for $j \geq 1$. This means that we need only consider the set $\Omega^{*}$ of nondecreasing sequences $\tau=\left(\tau_{0}, \tau_{1}, \tau_{2}, \ldots\right)$, or even the set $\Omega^{\prime}$ of strictly increasing sequences. The conditional density $\mu\left(\tau_{j+1} \mid \tau_{0}, \ldots, \tau_{j}\right)$ is assumed to be continuous with respect to $\left(\tau_{1}, \ldots, \tau_{j}\right)$, $0 \leq \tau_{1} \leq \tau_{2} \leq \ldots \leq \tau_{j} \leq \tau_{j+1}$, for each $\tau_{j+1}$. Moreover, the existence of integrable functions $\mu_{j+1}^{*}($.$) is assumed,$ such that, for all $\left(\tau_{0}, \ldots, \tau_{j}\right), \mu\left(\tau_{j+1} \mid \tau_{0}, \ldots, \tau_{j}\right) \leq \mu_{j+1}^{*}\left(\tau_{j+1}\right)$ a.e. For $\tau^{j}:=\left(\tau_{0}, \tau_{1}, \ldots, \tau_{j}\right)$, the conditional densities define simultaneous conditional densities $\mu\left(\tau_{j+1}, \ldots, \tau_{m} \mid \tau^{j}\right)\left(\mu\left(\tau_{1}, \ldots, \tau_{m} \mid \tau^{0}\right)=\mu\left(\tau_{1}, \ldots, \tau_{m}\right)\right)$, assumed to satisfy: for some $k_{*} \in(0,1)$, and some positive number $\Phi^{*}(t)$,

$$
\operatorname{Pr}\left[t \in\left(\tau_{m}, \tau_{m+1}\right] \mid \tau^{j}\right] \leq \Phi^{*}(t)\left(k_{*}\right)^{m-j} \text { for any given } t \in[0, \infty) .
$$

Assume $\Phi^{*}:=\sup _{t \in[0, T]} \Phi^{*}(t)<\infty$. Property (2.5), used for $j=0$, means that with probability 1 , the sequences $\left(\tau_{0}, \tau_{1}, \tau_{2}, \ldots\right)$ has the property that $\tau_{i} \rightarrow \infty$. The set of $\tau$ 's in $\Omega^{\prime}$ such that $\tau_{i} \rightarrow \infty$ is denoted $\Omega^{\prime \prime}$. Below, it is assumed that any $\tau$ belongs to $\Omega^{\prime \prime}$.

Let the term "nonanticipating function" mean a function $y(t, \tau)=y\left(t, \tau_{0}, \tau_{1}, \ldots\right)$ that for each given $t \in[0, T]$, depends only on $\tau_{i}$ 's $\leq t$. (Formally, we require $y\left(t, \tau_{0}^{\prime}, \tau_{1}^{\prime}, \ldots\right)=y\left(t, \tau_{0}, \tau_{1}, \ldots\right)$ if $\left\{i: \tau_{i}^{\prime} \leq t\right\}=\left\{i: \tau_{i} \leq t\right\}$ and 
$\tau_{i}^{\prime}=\tau_{i}$ for $\left.i \in\left\{i: \tau_{i} \leq t\right\}\right)$. Here, $y(.,$.$) is assumed to take values in a Euclidean space \bar{Y}$. Let $\mathcal{M}^{\text {nonant }}(J \times$ $\left.\Omega^{\prime \prime}, \bar{Y}\right)$ be the set of functions being nonanticipating and simultaneous measurable on each set $J \times \Omega_{i}, \Omega_{i}:=$ $\left\{\tau \in \Omega^{\prime}: \tau_{i} \leq T, \tau_{i+1}>T\right\}, i=1,2, \ldots{ }^{3}$.

Define $\Omega^{i}:=\left\{\tau^{i}: \tau \in \Omega_{i}\right\}$ and $U^{\prime}:=\left\{u(.,.) \in \mathcal{M}^{\text {nonant }}\left(J \times \Omega^{\prime \prime}, \mathbb{R}^{r}\right): u(t, \tau) \in U\right.$ for all $\left.(t, \tau)\right\}$. From now on, all control functions $u(t, \tau)$ belong to $U^{\prime}$, they are called admissible if in addition corresponding solutions on $[0, T]$ of (2.2) exist a.s., that satisfy (2.3) and (2.4) a.s., ("a.s." here taken to mean for all $\tau \in \Omega^{\prime \prime} \cap\left(\cup_{i}\left\{\Omega_{i} \backslash N_{i}\right\}\right)$ for some P-null sets $N_{i}$ in $\Omega_{i}$ ). For any given $u(.,.) \in U^{\prime}$ and for any given $\tau \in \Omega^{\prime \prime}$, the differential equation in (2.2) is an ordinary deterministic equation, and it is assumed that solutions on $[0, T]$ of $(2.2)$ are unique, for any given $u(.,.) \in U^{\prime}$.

As functions of $(t, \tau)$, for all $(x, u) f_{0}$ and $f$ are now assumed to be nonanticipating. Furthermore, $t \mapsto$ $f(t, x, u, \tau)$ and $t \rightarrow f_{0}(t, x, u, \tau)$ have one-sided limits at each point and is right continuous, and for each $i$, $f\left(t, x, u, \tau^{i}, T+1, T+2, \ldots\right)$ and $f_{0}\left(t, x, u, \tau^{i}, T+1, T+2, \ldots\right)$ are continuous in $\left\{\left(t, x, u, \tau^{i}\right): t \in J, x \in \mathbb{R}^{n}, u \in\right.$ $\left.U, \tau^{i} \in \Omega^{i}, \tau_{i} \leq t\right\}^{4},\left(\tau_{i}=\left(\tau^{i}\right)_{i}\right)$. Finally, $h^{*}$ is $C^{1}$ with bounded derivative. Let us call the above assumptions on $f_{0}, h^{*}$, and $f$ for the general assumptions. (These assumptions imply that e.g. $f$ can essentially be written as $f(t, x, u, \tau)=\sum_{i \geq 0} f^{i}\left(t, x, u, \tau^{i}\right) 1_{\left[\tau_{i}, \tau_{i+1}\right)}(t), \tau=\left(\tau_{0}, \tau_{1}, \ldots\right) \in \Omega^{\prime}$ for certain continuous functions $f^{i}\left(t, x, u, \tau^{i}\right)$, $i=0,1, \ldots)$.

The specific conditions needed in the first existence theorem are as follows:

there exists an admissible pair

$$
x(., .), u(., .),\left(x(., .)=x^{u(. .)}(., .)\right) \text {, thus }(x(., .), u(., .)) \text { satisfies }(2.2),(2.3), \text { and }(2.4), \text { with } u(., .) \text { in } U^{\prime} \text {, }
$$

$U$ is compact,

and

$$
N(t, x, \tau)=\left\{\left(f_{0}(t, x, u, \tau)+\gamma, f(t, x, u, \tau)\right): u \in U, \gamma \leq 0\right\} \text { is convex for all }(t, x, \tau) .
$$

Moreover, there exist positive numbers $K_{i}$ and positive continuous functions $r_{i}^{*}(t)$, and a number $\bar{k} \in\left(1,1 / k_{*}\right)$, (for $k_{*}$, see (2.5)) with $\sup K_{i} / \bar{k}^{i}<\infty$, such that (2.9) and (2.10) below hold.

$$
|f(t, x, u, \tau)| \leq K_{i},\left|f_{0}(t, x, u, \tau)\right| \leq K_{i} \text {, for all }(x, u, \tau) \in \operatorname{cl} B\left(x_{0}, r_{i}^{*}(t)\right) \times U \times \Omega^{\prime \prime}, \text { all } t \in\left(\tau_{i}, \tau_{i+1}\right) \cap J .
$$

For any control $u(.,.) \in U^{\prime}$ and any $\tau \in \Omega^{\prime \prime}$, any solution $t \mapsto x\left(t, \tau ; \tau_{i}, \bar{x}\right)$ on $\left[\tau_{i}, T\right]$ of $\dot{x}=f(t, x, u(t, \tau), \tau)$ starting at $\left(\tau_{i}, \bar{x}\right), \bar{x} \in \operatorname{cl} B\left(x_{0}, r_{i-1}^{*}\left(\tau_{i}\right)\right)$ is unique and satisfies, for $j \geq i \geq 1, x\left(t, \tau ; \tau_{i}, \bar{x}\right) \in \operatorname{cl} B\left(x_{0}, r_{j}^{*}(t)\right)$ for all $t \in\left[\tau_{j}, \tau_{j+1}\right] \cap J$. Moreover, a solution $x\left(t, \tau ; \tau_{0}, x_{0}\right), t \in[0, T]$, corresponding to any control in $U^{\prime}$ is unique and satisfies

$$
x\left(t, \tau ; \tau_{0}, x_{0}\right) \in \operatorname{cl} B\left(x_{0}, r_{j}^{*}(t)\right) \text { for all } t \in\left[\tau_{j}, \tau_{j+1}\right] \cap J, j \geq 0 .
$$

Theorem 2.1. If the general assumptions are satisfied, and (2.6)-(2.10) hold, then an optimal admissible control exists.

Proof. It suffices to consider the special case where $a x^{u}(T)$ is maximized, $a$ a fixed nonzero vector in $\mathbb{R}^{n}{ }^{5}$. For simplicity, let $x_{0}=0$. Define $\hat{\tau}^{k}=\min \left\{T, \tau_{k}\right\}$.

\footnotetext{
${ }^{3}$ These properties are essentially equivalent to progressive measurability with respect to the subfields $\Phi_{t}$ defined as follows: let $\Phi_{t}, t \in[0, T]$, be the $\sigma$-algebra generated by sets of the form $A=A_{B, i}:=\left\{\tau:=\left(\tau_{1}, \tau_{2}, \ldots\right) \in \Omega^{\prime \prime}: \tau_{i} \in B\right\}$, where $B$ is either a measurable set in $[0, t]$, or $B=(t, \infty), i \in\{1,2, \ldots\}$. A probability measure $P$, corresponding to the conditional densities $\dot{\mu}\left(\tau_{i+1} \mid \tau^{i}\right)$, is defined on $\left(\Omega^{\prime \prime}, \Phi\right), \Phi:=\Phi_{T}$.

${ }^{4}$ Here we can replace $t \in J$, by $t \in J \backslash\left\{a_{1}, \ldots, a_{m}\right\}$, where $a_{i}$ are fixed numbers. In fact, concerning the dependence on $t$, much weaker conditions are actually needed, the continuity conditions above were chosen in order to be able to refer to the classical, simple results of Cesari [2], Sections 9.2, 9.3.

${ }^{5}$ In case of the criterion (2.1), two addition states $x_{0}$ and $x_{n+1}$ and an auxiliary control $u_{0} \in[0,1]$, can be introduced, with $\dot{x}_{0}=f^{0}\left(t, x, u_{0}, u, \tau\right):=u_{0} \cdot\left(f_{0}(t, x, u, \tau)+K_{i}\right)-K_{i}$, for $t \in\left(\tau_{i}, \tau_{i+1}\right), x_{0}(0)=0, \dot{x}_{n+1}=f^{n+1}(t, x, u, \tau):=h_{x}^{*}(x(t, \tau)) f(t, x, u, \tau)$, $x_{n+1}(0)=h^{*}\left(x_{0}\right)$, in which case the criterion in (2.1) equals $a \cdot\left(x_{0}^{u}(T), x^{u}(T), x_{n+1}^{u}(T)\right), a=\left(1,0_{n}, 1\right), 0_{n}$ the origin in $\left.\mathbb{R}^{n}\right)$. (Concavity of $\left\{\left(f^{0}\left(t, x, u_{0}, u, \tau\right), f(t, x, u, \tau), f^{n+1}(t, x, u, \tau)\right):\left(u_{0}, u\right) \in[0,1] \times U\right\}$ then holds. Also $f^{0} \leq f_{0}$, with equality if $\left.u_{0}=1\right)$.
} 
Outline of proof. In proofs of existence theorems in the stationary case, existence results from deterministic control theory is combined with proving certain smoothness properties of the optimal value function in order to obtain an existence proof, (see e.g. Davis [3]). Below, such an argument is recursively repeated in the nonautonomous case.

The central part of the proof of the theorem is the following: let $V^{k, \infty}\left(x, \tau_{k}\right)$ be the supremum over "admissible" controls of the conditional expectation of the criterion $a x^{u}(T, \tau)$ given $\tau^{k}$ and given that the solutions start at $\left(\tau_{k}, x\right)$, i.e. $\tau_{k}$ has just occurred, and the state at which we start at that time is $x$. (Here admissible means the existence of solutions satisfying the terminal conditions (2.3) and (2.4). If no such controls exists, we let the supremum be equal to $-\infty$ ). Then, as shown below, a relationship similar to the optimality equation in dynamic programming holds:

$$
V^{k, \infty}\left(x, \tau^{k}\right)=\sup _{u} E_{\tau_{k+1}}\left[a \int_{\hat{\tau}^{k}}^{\hat{\tau}^{k+1}} f\left(s, x^{u}(s), u(s), \tau\right) \mathrm{d} s+V^{k+1, \infty}\left(x^{u}\left(\hat{\tau}^{k+1}\right), \tau^{k+1}\right) \mid \tau^{k}\right],
$$

( $E_{\tau_{k+1}}$ means expectation with respect to $\tau_{k+1}$, i.e., with $\tau_{k+1}$ as integration variable). Here the supremum is taken over all deterministic functions $u($.$) for which the corresponding deterministic solutions x^{u}(t)$ satisfy the terminal conditions in case $\operatorname{Pr}\left[\tau_{k+1}>T \mid \tau^{k}\right]>0$, and start at $\left(\hat{\tau}_{k}, x\right)$. Generally, $V^{k, \infty}\left(x, \tau^{k}\right)=0$ if $\tau_{k} \geq T$ $\Leftrightarrow \hat{\tau}^{k}=T$. Let us then construct the optimal controls by induction. (Below, this construction is repeated, with more detailed arguments.) By existence theorems for deterministic control (more precisely Remark 2.3 below), there exists a control $u_{0}(t)=u_{0, \tau^{0}}(t)$ with corresponding solution $x_{0, \tau^{0}}(t),\left(x_{0, \tau^{0}}(0)=x_{0}\right)$, yielding the supremum in (2.11) for $k=0$, and satisfying the terminal conditions if $\operatorname{Pr}\left[\tau_{1}>T\right]>0$. By induction, for each $\tau^{k-1}$ such that $\tau_{k-1} \in\left(\tau_{k-2}, T\right)$, assume $u_{k-1, \tau^{k-1}}(t)$ defined, with corresponding solution $x_{k-1, \tau^{k-1}}(t)$ yielding supremum in (2.11) and satisfying $x_{k-1, \tau^{k-1}}\left(\tau_{k-1}\right)=x_{k-2, \tau^{k-2}}\left(\tau_{k-1}\right)$ and the terminal conditions if $\operatorname{Pr}\left[\tau_{k}>T \mid \tau^{k-1}\right]>0$. By existence theorems in deterministic control theory, (Rem. 2.3 below), for each $\tau^{k}$ such that $\tau_{k} \in\left(\tau_{k-1}, T\right)$, there exists a control function $u_{k, \tau^{k}}(t)$ with corresponding solution $x_{k, \tau^{k}}(t)$, starting at $\left(\tau_{k}, x_{k-1, \tau^{k-1}}\left(\tau_{k}\right)\right)$ and satisfying the terminal conditions if $\operatorname{Pr}\left[\tau_{k+1}>T \mid \tau^{k}\right]>0$, that yields the supremum in (2.11). So $u_{k, \tau^{k}}(t)$ exists for all $k$. Using (2.11) for $k=0,1,2, \ldots$, for any given $k$,

$$
V^{0, \infty}(0,0)=E\left[a \sum_{j=0}^{k} \int_{\hat{\tau}^{j}}^{\hat{\tau}^{j+1}} f\left(s, x_{j, \tau^{j}}(s), u_{j, \tau^{j}}(s), \tau\right) \mathrm{d} s+V^{k+1, \infty}\left(x_{k, \tau^{k}}\left(\hat{\tau}^{k+1}\right), \tau^{k+1}\right)\right]
$$

When $k \rightarrow \infty$, as $E\left[V^{k+1, \infty}\left(x_{k, \tau_{k}}\left(\hat{\tau}^{k+1}\right), \tau^{k+1}\right)\right] \rightarrow 0$, we get $V^{0, \infty}(0,0)=E\left[a \sum_{j=0}^{\infty} \int_{\hat{\tau}^{j}}^{\hat{\tau}^{j+1}}\right.$ $\left.f\left(s, x_{j, \tau^{j}}(s), u_{j, \tau^{j}}(s), \tau\right) \mathrm{d} s\right]$. Hence, the pair $\left(x^{*}(t, \tau), u^{*}(t, \tau)\right)$ defined by $\left(x^{*}(t, \tau), u^{*}(t, \tau)\right)=\left(x_{k, \tau^{k}}(t), u_{k, \tau^{k}}(t)\right)$ for $t \in\left(\tau_{k}, \tau_{k+1}\right) \cap[0, T]$ is optimal. (It is admissible because if $T \in\left(\tau_{k}, \tau_{k+1}\right)$, then $x^{*}(T, \tau)=x_{k, \tau^{*}}(T)$ satisfies the terminal conditions if $\operatorname{Pr}\left[\tau_{k+1}>T \mid \tau^{k}\right]>0$, hence $x^{*}(T, \tau)$ a.s. satisfies these conditions if $\left.T \in\left(\tau_{k}, \tau_{k+1}\right)\right)$.

Detailed proof. First we need a proposition, with a related remark. The proposition is an existence result from deterministic control theory, contained in results appearing in Chapters 8-10 in Cesari [2]. For convenience of the reader a brief proof is included, making use of wellknown elementary properties taken from deterministic existence proofs.

Consider the following deterministic system: define $\tilde{U}$ to be the set of measurable functions from $[0, T]$ into $U$. Let $f(t, x, u): J \times \mathbb{R}^{n} \times U \rightarrow \mathbb{R}^{n}$ be continuous in $S \times U, S$ a compact set in $J \times \mathbb{R}^{n}$. Let $\left(t_{0}, x_{0}\right) \in S$, and define the set $A\left(t_{0}, x_{0}\right)$ to consist of all pairs $x(),. u(),. u(.) \in \tilde{U}$ that satisfy $(t, x(t)) \in S$ for all $t \in\left[t_{0}, T\right]$ and the following differential equation with side conditions:

$$
\text { for a.e. } t \in\left[t_{0}, T\right], \dot{x}=f(t, x, u(t)), x\left(t_{0}\right)=x_{0}, x(T) \in B \text {, }
$$


where $B$ is a closed set in $\mathbb{R}^{n}$. Let $h(x): \mathbb{R}^{n} \rightarrow \mathbb{R} \cup\{-\infty\}$ be upper semicontinuous, (abbreviated usc), and let $g(t, x): J \times \mathbb{R}^{n} \rightarrow \mathbb{R} \cup\{-\infty\}$ be usc in $S$. Consider the problem

$$
\max _{x(.), u(.)}\left[\int_{t_{0}}^{T} g(t, x(t)) \mathrm{d} t+h(x(T))\right] .
$$

Assume that $U$ is compact, that $f(t, x, U)$ is convex for all $(t, x) \in S$, that there exist a positive integrable function $\psi(t)$ and a positive number $K$ such that $|f(t, x, u)| \leq K$ and $g(t, x) \leq \psi(t)$ for all $(t, x, u) \in S \times U$.

Define the set $C \subset S$ to be the set of points $\left(t_{0}, x_{0}\right)$ in $S$ for which a pair $\left.(x),. u().\right), u(.) \in \tilde{U}$ exists, satisfying $(t, x(t)) \in S$ for all $t \in\left[t_{0}, T\right]$ and $(2.12)$. Let $V\left(t_{0}, x_{0}\right):=\sup _{(x(.), u(.)) \in A\left(t_{0}, x_{0}\right)} V^{x(.), u(.)}$, where $V^{x(.), u(.)}\left(t_{0}, x_{0}\right)=\int_{t_{0}}^{T} g(t, x(t)) \mathrm{d} t+h(x(T))$, and where $\left(t_{0}, x_{0}\right)$ belongs to $C$. The following result holds for this system:

Proposition 2.2. For any $\left(t_{0}, x_{0}\right) \in C$, an optimal pair $(x(),. u()),. u(.) \in \tilde{U}$ exists, satisfying (2.12) and $(t, x(t)) \in S$, (perhaps the corresponding value of the criterion is $-\infty)$. Moreover, $C$ is closed, and $V\left(t_{0}, x_{0}\right)$ is usc in $\left(t_{0}, x_{0}\right) \in C$.

Proof of Proposition 2.2. For $k=1,2, \ldots$, when $k \rightarrow \infty$, let $\left(t_{0}^{k}, x_{0}^{k}\right) \rightarrow\left(t_{0}, x_{0}\right),\left(t_{0}, x_{0}\right),\left(t_{0}^{k}, t_{0}^{k}\right) \in S$. Let $I_{k}:=$ $\left[t_{0}^{k}, T\right], I=\left[t_{0}, T\right]$. Assume (A) that a sequence $\left(x^{k}(),. u^{k}().\right)$ is given that satisfies $(2.12)$ for $\left(t_{0}, x_{0}\right)=\left(t_{0}^{k}, x_{0}^{k}\right)$

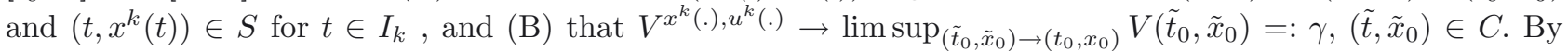
standard arguments, (see e.g. Cesari [2], Sects. 9.2, 9.3), there exists a subsequence $x^{k_{j}}($.$) , a control function$ $u^{*}(.) \in \tilde{U}$, and a continuous function $x^{*}($.$) such that \sup _{t \in I_{k_{j}} \cap I}\left|x^{k_{j}}(t)-x^{*}(t)\right| \rightarrow 0$, and such that $\left(x^{*}(),. u^{*}().\right)$ satisfies (2.12) and $\left(t, x^{*}(t)\right) \in S$. By slight misuse of notation, by upper integrable boundedness of $g$ and Fatou's lemma,

$$
\begin{aligned}
\gamma & =\lim \sup _{j}\left[\int_{t_{0}^{k_{j}}}^{T} g\left(t, x^{k_{j}}(t)\right) \mathrm{d} t+h\left(x^{k_{j}}(T)\right)\right] \\
& \leq \int_{J}\left(\lim \sup _{j} g\left(t, x^{k_{j}}(t)\right) 1_{I_{k_{j}}}\right) \mathrm{d} t+\lim \sup _{j} h\left(x^{k_{j}}(T)\right) \\
& \leq \int_{J} g\left(t, x^{*}(t)\right) 1_{I} \mathrm{~d} t+h\left(x^{*}(T)\right) .
\end{aligned}
$$

Hence, $V\left(t_{0}, x_{0}\right)$ is usc. Dropping the assumption (B), we get that $C$ is closed. If all $t_{0}^{k}=t_{0}, x_{0}^{k}=x_{0}$, and we (instead of $(\mathrm{B})$ ), assume that $V^{x^{k}(.), u^{k}(.)} \rightarrow V\left(t_{0}, x_{0}\right)$, then the above arguments give that $V\left(t_{0}, x_{0}\right) \leq$ $\int_{J} g\left(t, x^{*}(t)\right) 1_{I} \mathrm{~d} t+h\left(x^{*}(T)\right)$, hence $\left(x^{*}(),. u^{*}().\right)$ is optimal.

If $V$ is defined to be equal to $-\infty$, for $\left(t_{0}, x_{0}\right) \in S \backslash C$, then $V$ is usc in $S$.

Remark 2.3. In Proposition 2.2, assume that $f, g$ and $h$ contain an additional parameter $y \in \mathbb{R}^{\hat{\imath}}, f=$ $f(t, x, u, y), g=g(t, x, y), h=h(x, y)$, and that $(2.12)$ is augmented by:

$$
\dot{y}=0, y\left(t_{0}\right)=y_{0}, y(T) \text { free. }
$$

Assume that the conditions in Proposition 2.2 are satisfied in this augmented system, (for $x$ replaced by $(x, y$ ), hence for $(t, x, y)$ in a compact subset $S)$. The conclusions of Proposition 2.2 hold for this system, in particular, the value function $V\left(t_{0}, x_{0}, y_{0}\right)$ in this system is usc in $\left(t_{0}, x_{0}, y_{0}\right) \in S$.

\section{Continued proof of Theorem 2.1.}

Let $B^{*}=\left\{x \in \mathbb{R}^{n}: x^{i}=\bar{x}^{i}, i=1, \ldots, n_{1}, x^{i} \geq \bar{x}^{i}, i=n_{1}+1, \ldots, n_{2}\right\}$. For any deterministic control $u(.) \in \tilde{U}$ and for any $\tau \in \Omega_{k}$, write $x(t)=x^{u}\left(t, \tau ; \tau_{k}, x\right)$ for the solution $x(t)$ on $\left[\tau_{k}, T\right]$ that satisfies $\dot{x}=f(t, x, u(t), \tau)$, 
$x\left(\tau_{k}\right)=x$. Let $U^{k, x, \tau}, \tau \in \Omega_{k}$, be the set of deterministic controls in $\tilde{U}$ for which there exists a solution $x^{u}\left(t, \tau ; \tau_{k}, x\right)$ of the differential equation in $(2.2)$ on $\left[\tau_{k}, T\right]$ that satisfies $x^{u}\left(T, \tau ; \tau_{k}, x\right) \in B^{*}$ if $\operatorname{Pr}\left[\tau_{k+1}>\right.$ $\left.T \mid \tau^{k}\right]>0$, with no terminal condition on $x^{u}\left(T, \tau ; \tau_{k}, x\right)$ if this inequality fails. Below, we will need the following definitions: let $l_{k}\left(\tau^{k}\right):=\int_{T}^{\infty} \mu\left(\tau_{k+1} \mid \tau^{k}\right) \mathrm{d} \tau_{k+1}$, and let

$$
B_{k}=\left\{\left(x, \tau^{k}\right) \in \mathbb{R}^{n} \times \Omega^{k}:\left(x^{i}-\bar{x}^{i}\right) l_{k}\left(\tau^{k}\right)=0, i=1, \ldots, n_{1},\left(x^{i}-\bar{x}^{i}\right) l_{k}\left(\tau^{k}\right) \geq 0, i=n_{1}+1, \ldots, n_{2}\right\} .
$$

(By continuity of $l_{k}(),. B_{k}$ is relatively closed in $\mathbb{R}^{n} \times \Omega^{k}$ ). For

$$
\begin{gathered}
u(.) \in U^{N, x, \tau}, \tau \in \Omega_{N}, \text { let } V_{u}^{N, N}\left(x, \tau^{N}\right):=E_{\tau_{N+1}}\left[a \int_{\hat{\tau}^{N}}^{T} 1_{[T, \infty)}\left(\tau_{N+1}\right) f\left(\check{s}, x^{u}\left(\check{s}, \tau ; \tau_{N}, x\right), u(\check{s}), \tau\right) \mathrm{d} \check{s} \mid \tau^{N}\right], \\
V^{N, N}\left(x, \tau^{N}\right)=\sup _{u \in U^{N, x, \tau}} V_{u}^{N, N}\left(x, \tau^{N}\right) .
\end{gathered}
$$

For $k \leq N$, by backwards induction, for $u(.) \in U^{k-1, x, \tau}, \tau \in \Omega_{k-1}$, define

$$
\begin{gathered}
V_{u}^{k-1, N}\left(x, \tau^{k-1}\right):=E_{\tau_{k}}\left[a \int_{\hat{\tau}^{k-1}}^{\hat{\tau}^{k}} f\left(\check{s}, x^{u}\left(\check{s}, \tau ; \tau_{k-1}, x\right), u(\check{s}), \tau\right) \mathrm{d} \check{s}+V^{k, N}\left(x^{u}\left(\hat{\tau}^{k}, \tau ; \tau_{k-1}, x\right), \tau^{k}\right) \mid \tau^{k-1}\right], \\
V^{k-1, N}\left(x, \tau^{k-1}\right):=\sup _{u \in U^{k-1, x, \tau}} V^{k-1, N}\left(x ; \tau^{k-1}\right) .
\end{gathered}
$$

All the time, the convention is used that when taking supremum over an empty set, we get $-\infty$.

Define $B^{k}:=\left\{\left(x, \tau^{k}\right): x \in \operatorname{cl} B\left(0, r_{k-1}^{*}\left(\tau_{k}\right)\right), \tau^{k} \in \Omega^{k}\right\}$. With the "specifications"

$$
S=\left\{(t, x): t \in\left[\tau_{N}, T\right], x \in \operatorname{cl} B\left(0, r_{N}^{*}(t)\right)\right\} \times \Omega^{N} \times \operatorname{cl} B\left(0, r_{N-1}^{*}\left(\tau_{N}\right)\right),
$$

$B=B_{N} \times \mathbb{R}^{n}, g=0, h(x, y)=a\left(x-y_{2}\right) \operatorname{Pr}\left[\tau_{N+1}>T \mid y_{1}\right], y=\left(y_{1}, y_{2}\right), y_{1}=\tau^{N}, y_{2} \in \mathbb{R}^{n}, \dot{x}(s)=f\left(s, x, u(s), y_{1}\right)$, $t_{0}=\tau_{N}, x\left(\tau_{N}\right)=\tilde{x}, y_{2}\left(\tau_{N}\right)=\tilde{x}$, Remark 2.3 yields that $V^{N, N}\left(\tilde{x}, \tau^{N}\right)$ is usc in $\left(\tilde{x}, \tau^{N}\right) \in B^{N},\left(\left(\tilde{x}, \tau^{N}\right) \in B^{N} \Rightarrow\right.$ $\left(\tau_{N}, \tilde{x}, \tau^{N}, \tilde{x}\right) \in S$, by $\left.(2.10)\right)$. Now, $S$ as here defined is not compact, nor is $B_{N}$ closed, but for any $\left(t, x, \tau^{N}, y_{2}\right)$ in $S$ we can replace $\Omega^{N}$ in the definitions of $S$ and $B_{N}$ by a compact neighborhood $\Omega^{*}$ of $\tau^{N}$ in $\Omega^{N}$, and obtain compactness, respectively closedness, of these redefined sets, and in particular usc in the redefined set $S$, and so in the original set $S$.

By backwards induction, assume that $\left(x, \tau^{k}\right) \rightarrow V^{k, N}\left(x, \tau^{k}\right)$ is usc on $B^{k}$. Letting $y=\left(y_{1}, y_{2}\right), y_{2} \in \mathbb{R}^{n}$, $y_{1}=\tau^{k-1}, g(t, x, y)=\left[a\left(x-y_{2}\right)+V^{k, N}\left(x, y_{1}, t\right)\right] \mu\left(t \mid y_{1}\right)\left(\tau^{k}=\left(y_{1}, t\right)\right), h(x, y)=a\left(x-y_{2}\right) \operatorname{Pr}\left[\tau_{k}>T \mid y_{1}\right]$, $B=B_{k-1} \times \mathbb{R}^{n}, \dot{x}(s)=f\left(s, x, u(s), y_{1}\right), t_{0}=\tau_{k-1}, x\left(\tau_{k-1}\right)=\tilde{x}, y_{2}\left(\tau_{k-1}\right)=\tilde{x}$ and $S:=\{(t, x): t \in$ $\left.\left[\tau_{k-1}, T\right], x \in \operatorname{cl} B\left(0, r_{k-1}^{*}(t)\right)\right\} \times \Omega^{k-1} \times \operatorname{cl} B\left(0, r_{k-2}^{*}\left(\tau_{k-1}\right)\right)$, Remark 2.3 yields that $\left(\tilde{x}, \tau^{k-1}\right) \mapsto V^{k-1, N}\left(\tilde{x}, \tau^{k-1}\right)$ is usc in $\left(\tilde{x}, \tau^{k-1}\right) \in B^{k-1}$. (When $\left(\tilde{x}, \tau^{k-1}\right)$ belongs to $B^{k-1}$, then automatically $\left(t, x^{u}\left(t, \tau ; \tau_{k-1}, \tilde{x}\right), \tau^{k-1}, \tilde{x}\right)$ belongs to $S$, by (2.10)). The set $S$ as here defined is not compact, nor is $B_{k-1}$ closed, but for any $\left(t, x, \tau^{k-1}, y_{2}\right)$ in $S$ we can replace $\Omega^{k-1}$ in the definitions of $S$ and $B_{k-1}$ by a compact neighborhood $\Omega^{*}$ of $\tau^{k-1}$ in $\Omega^{k-1}$, and obtain compactness, respectively closedness, of these redefined sets. In particular, usc holds in the redefined set $S$, and so in the original set $S$.

For any given admissible control $u(t, \tau) \in U^{\prime}$ with corresponding solution $x^{u}(t, \tau)$, let us prove the following inequality by backwards induction on $k$.

$$
\begin{aligned}
E\left[a x^{u}(T, \tau)\right] \leq & E\left[\sum_{0 \leq j \leq k-1} \int_{\hat{\tau}^{j}}^{\hat{\tau}^{j+1}} a f\left(s, x^{u}(s, \tau), u(s, \tau), \tau\right) \mathrm{d} s\right] \\
& +E\left[V^{k, N}\left(x^{u}\left(\hat{\tau}^{k}, \tau\right), \tau^{k}\right)\right]+E\left[\sigma_{N+1}\left(\tau^{N+1}\right)\right], k \leq N,
\end{aligned}
$$


where $\sigma_{N+1}\left(\tau^{N+1}\right):=E\left[\sigma^{N+1}(\tau) \mid \tau^{N+1}\right]$,

$$
\sigma^{N+1}(\tau):=\sum_{N+1 \leq j<\infty} \int_{\hat{\tau}^{j}}^{\hat{\tau}^{j+1}} a f\left(s, x^{u}(s, \tau), u(s, \tau), \tau\right) \mathrm{d} s+1_{[0, T]}\left(\tau_{N+1}\right) \int_{\hat{\tau}^{N}}^{\hat{\tau}^{N+1}} a f\left(s, x^{u}(s, \tau), u(s, \tau), \tau\right) \mathrm{d} s .
$$

Proof of (2.18). Let us first show that, for all $\tau \in \Omega_{k}$, a.s., $u(t, \tau)$ belongs to $U^{k, x^{u}\left(\tau_{k}, \tau\right), \tau}$. Let the deterministic $\hat{u}($.$) equal u(t, \tau)$, for $t \in\left[\tau_{k}, T\right], \tau_{k+1} \geq T$. Since $x^{u}(T, \tau) \in B^{*}$ a.s., then for all $\tau \in \Omega_{k}$, a.s., if $\operatorname{Pr}\left[\tau_{k+1}>\right.$ $\left.T \mid \tau^{k}\right]>0$, we have that $x^{\hat{u}}\left(T, \tau ; \tau_{k}, x^{u}\left(\tau_{k}, \tau\right)\right)=x^{u}(T, \tau) \in B^{*}$. Then, for all $\tau^{k}$, a.s., $\hat{u}(.) \in U^{k, x^{u}\left(\tau_{k}, \tau\right), \tau}$ and, evidently, the assertion follows.

Now, using $(2.14),(2.15)$, a.s. in $\tau \in \Omega_{N}, V^{N, N}\left(x^{u}\left(\hat{\tau}^{N}, \tau\right), \tau^{N}\right) \geq E\left[1_{(T, \infty)}\left(\tau_{N+1}\right) \int_{\hat{\tau}^{N}}^{\hat{\tau}^{N+1}} a f\left(s, x^{u}(s, \tau)\right.\right.$, $\left.u(s, \tau), \tau) \mathrm{d} s \mid \tau^{N}\right]$, since, a.s., $\hat{u}(.) \in U^{N, x^{u}\left(\tau_{N}, \tau\right), \tau}$, where $\hat{u}($.$) is the deterministic control that equals u(t, \tau)$, for $t \in\left[\tau_{N}, T\right]$ when $\tau_{N+1} \in[T, \infty)$. Furthermore, we have

$$
\begin{aligned}
E\left[a x^{u}(T, \tau) \mid \tau^{N}\right]= & E\left[\sum_{0 \leq j<\infty} \int_{\hat{\tau}^{j}}^{\hat{\tau}^{j+1}} a f\left(s, x^{u}(s, \tau), u(s, \tau), \tau\right) \mathrm{d} s \mid \tau^{N}\right] \\
= & E\left[\sum_{0 \leq j<N-1} \int_{\hat{\tau}^{j}}^{\hat{\tau}^{j+1}} a f\left(s, x^{u}(s, \tau), u(s, \tau), \tau\right) \mathrm{d} s \mid \tau^{N}\right] \\
& +E\left[\sum_{N+1 \leq j<\infty} \int_{\hat{\tau}^{j}}^{\hat{\tau}^{j+1}} a f\left(s, x^{u}(s, \tau), u(s, \tau), \tau\right) \mathrm{d} s \mid \tau^{N}\right] \\
& +E\left[1_{[0, T]}\left(\tau_{N+1}\right) \int_{\hat{\tau}^{N}}^{\hat{\tau}^{N+1}} a f\left(s, x^{u}(s, \tau), u(s, \tau), \tau\right) \mathrm{d} s \mid \tau^{N}\right] \\
& +E\left[1_{(T, \infty)}\left(\tau_{N+1}\right) \int_{\hat{\tau}^{N}}^{\hat{\tau}^{N+1}} a f\left(s, x^{u}(s, \tau), u(s, \tau), \tau\right) \mathrm{d} s \mid \tau^{N}\right] \\
= & E\left[\sum_{0 \leq j<N-1} \int_{\hat{\tau}^{j}}^{\hat{\tau}^{j+1}} a f\left(s, x^{u}(s, \tau), u(s, \tau), \tau\right) \mathrm{d} s \mid \tau^{N}\right]+E\left[\sigma_{N+1}\left(\tau_{N+1}\right) \mid \tau^{N}\right] \\
& +E\left[1_{(T, \infty)}\left(\tau_{N+1}\right) \int_{\hat{\tau}^{N}}^{\hat{\tau}^{N+1}} a f\left(s, x^{u}(s, \tau), u(s, \tau), \tau\right) \mathrm{d} s \mid \tau^{N}\right] .
\end{aligned}
$$

Replacing the last term by the greater term $V^{N, N}\left(x^{u}\left(\hat{\tau}^{N}, \tau\right), \tau^{N}\right)$, (see the last inequality), we get, for $\tau \in \Omega_{N}$, that, a.s.,

$$
\begin{aligned}
E\left[a x^{u}(T, \tau) \mid \tau^{N}\right] \leq & E\left[\sum_{0 \leq j \leq N-1} \int_{\hat{\tau}^{j}}^{\hat{\tau}^{j+1}} a f\left(s, x^{u}(s, \tau), u(s, \tau), \tau\right) \mathrm{d} s \mid \tau^{N}\right] \\
& +V^{N, N}\left(x^{u}\left(\hat{\tau}^{N}, \tau\right), \tau^{N}\right)+E\left[\sigma_{N+1}\left(\tau^{N+1}\right) \mid \tau^{N}\right] .
\end{aligned}
$$

Using that $V^{N, N}\left(x^{u}\left(\hat{\tau}^{N}, \tau\right), \tau^{N}\right)$ vanishes when $\tau_{N} \geq T$, (in which case the inequality is an equality), by taking expectations on both sides, (2.18) follows for $k=N$. Now, for $\tau \in \Omega_{k}$, a.s. $u(t, \tau) \in U^{k, x^{u}\left(\tau_{k}, \tau\right), \tau}$ for $\tau_{k+1}>T$. Then evidently, for all $\tau \in \Omega_{k}$, a.s.,

$$
V^{k, N}\left(x^{u}\left(\hat{\tau}^{k}, \tau\right), \tau^{k}\right) \geq E\left[a \int_{\hat{\tau}^{k}}^{\hat{\tau}^{k+1}} a f\left(s, x^{u}(s, \tau), u(s, \tau), \tau\right) \mathrm{d} s+V^{k+1, N}\left(x^{u}\left(\hat{\tau}^{k+1}, \tau\right), \tau^{k+1}\right) \mid \tau^{k}\right] .
$$


In fact, (2.19) holds for all $\tau \in \Omega^{\prime \prime}$ a.s., since both sides of (2.19) are zero if $\tau_{k}>T$. Assume now that (2.18) holds for $k$ replaced by $k+1, k+1 \leq N$, and let us prove (2.18) as written. The induction hypothesis implies the first inequality below, and (2.19) implies the second one:

$$
\begin{aligned}
E\left[a x^{u}(T)\right] & \leq E\left[\sum_{0 \leq j \leq k-1} \int_{\hat{\tau}^{j}}^{\hat{\tau}^{j+1}} a f\left(s, x^{u}(s, \tau), u(s, \tau), \tau\right) \mathrm{d} s\right] \\
& +E\left[E\left[\int_{\hat{\tau}^{k}}^{\hat{\tau}^{k+1}} a f\left(s, x^{u}(s, \tau), u(s, \tau), \tau\right) \mathrm{d} s \mid \tau^{k}\right]\right] \\
& +E\left[E\left[V^{k+1, N}\left(x^{u}\left(\hat{\tau}^{k+1}, \tau\right), \tau^{k+1}\right) \mid \tau^{k}\right]\right] \\
& +E\left[\sigma_{N+1}\left(\tau^{N+1}\right)\right] \\
& \leq E\left[\sum_{0 \leq j \leq k-1} \int_{\hat{\tau}^{j}} \int^{\hat{\tau}^{j+1}} a f\left(s, x^{u}(s, \tau), u(s, \tau), \tau\right) \mathrm{d} s\right] \\
& +E\left[V^{k, N}\left(x^{u}\left(\hat{\tau}^{k}, \tau\right), \tau^{k}\right)\right]+E\left[\sigma_{N+1}\left(\tau^{N+1}\right)\right]
\end{aligned}
$$

So (2.18) has been proved by induction.

Proof of (2.11) (i.e. (2.26) below). For $u \in \tilde{U},\left(x, \tau^{N}\right) \in B^{N}$, define

$$
\hat{V}_{u}^{N, N}\left(x, \tau^{N}\right):=E\left[\int_{\hat{\tau}^{N}}^{\hat{\tau}^{N+1}} a f\left(s, x^{u}\left(s, \tau^{N} ; \tau_{N}, x\right), u(s), \tau\right) \mathrm{d} s \mid \tau^{N}\right] .
$$

Also, define $\hat{K}_{i}=|a| K_{i}$, (for $K_{i}$ see $\left.(2.9)\right)$. For any $\left(x, \tau^{N}\right) \in B^{N}$, note that

$$
\left|V_{u}^{N, N}\left(x, \tau^{N}\right)-\hat{V}_{u}^{N, N}\left(x, \tau^{N}\right)\right| \leq E\left[T \hat{K}_{N} 1_{[0, T]}\left(\tau_{N+1}\right) \mid \tau^{N}\right] .
$$

Similarly, for any $\left(x, \tau^{N+1}\right) \in B^{N+1}$,

$$
\left|V_{u}^{N+1, N+1}\left(x, \tau^{N+1}\right)-\hat{V}_{u}^{N+1, N+1}\left(x, \tau^{N+1}\right)\right| \leq E\left[T \hat{K}_{N+1} 1_{[0, T]}\left(\tau_{N+2}\right) \mid \tau^{N+1}\right] .
$$

Also,

$$
\begin{gathered}
\left|V_{u}^{N+1, N+1}\left(x, \tau^{N+1}\right)\right| \leq T \hat{K}_{N+1} 1_{[0, T]}\left(\tau_{N+1}\right), \\
\left(V_{u}^{N+1, N+1} \text { vanishes if } \tau_{N+1}>T\right), \text { so } V^{N+1, N+1}\left(x, \tau^{N+1}\right) \leq T \hat{K}_{N+1}\left[1_{[0, T]}\left(\tau_{N+1}\right)\right],
\end{gathered}
$$

and we also have

$$
V^{N+1, N+1}\left(x, \tau^{N+1}\right) \geq-T \hat{K}_{N+1}\left[1_{[0, T]}\left(\tau_{N+1}\right)\right]
$$

if $V^{N+1, N+1}\left(x, \tau^{N+1}\right)$ is finite $\left(\Leftrightarrow U^{N+1, x, \tau} \neq \emptyset\right)$. Hence, if $V^{N+1, N+1}\left(x, \tau^{N+1}\right)$ is finite, then, for $\left(x, \tau^{N+1}\right) \in$ $B^{N+1}$,

$$
\left|V^{N+1, N+1}\left(x, \tau^{N+1}\right)\right| \leq T \hat{K}_{N+1}\left[1_{[0, T]}\left(\tau_{N+1}\right)\right] .
$$

By $(2.16)$, for $\left(x, \tau^{N}\right) \in B^{N}$,

$$
V_{u}^{N, N+1}\left(x, \tau^{N}\right)=\hat{V}_{u}^{N, N}\left(x, \tau^{N}\right)+E\left[V^{N+1, N+1}\left(x^{u}\left(\hat{\tau}^{N+1}, \tau ; \tau_{N}, x\right), \tau^{N+1}\right) \mid \tau^{N}\right],
$$


so for $\beta\left(x, \tau^{N}\right):=E\left[V^{N+1, N+1}\left(x^{u}\left(\hat{\tau}^{N+1}, \tau ; \tau_{N}, x\right), \tau^{N+1}\right) \mid \tau^{N}\right]$, if $\beta\left(x, \tau^{N}\right)$ is finite, then

$$
\begin{aligned}
\left|V_{u}^{N, N+1}\left(x, \tau^{N}\right)-V_{u}^{N, N}\left(x, \tau^{N}\right)\right|= & \mid V_{u}^{N, N+1}\left(x, \tau^{N}\right)-\hat{V}_{u}^{N, N}\left(x, \tau^{N}\right) \\
& +\hat{V}_{u}^{N, N}\left(x, \tau^{N}\right)-V_{u}^{N, N}\left(x, \tau^{N}\right) \mid \\
= & \left|\beta\left(x, \tau^{N}\right)+\hat{V}_{u}^{N, N}\left(x, \tau^{N}\right)-V_{u}^{N, N}\left(x, \tau^{N}\right)\right| \\
\leq & \left|\beta\left(x, \tau^{N}\right)\right|+E\left[T \hat{K}_{N} 1_{[0, T]}\left(\tau_{N+1}\right) \mid \tau^{N}\right] \\
\leq & E\left[\left(T \hat{K}_{N}+T \hat{K}_{N+1}\right) 1_{[0, T]}\left(\tau_{N+1}\right) \mid \tau^{N}\right] \\
= & : \alpha\left(\tau^{N}\right) .
\end{aligned}
$$

Hence, $V_{u}^{N, N+1}\left(x, \tau^{N}\right) \leq V_{u}^{N, N}\left(x, \tau^{N}\right)+\alpha\left(\tau^{N}\right)$, (which also holds if $V_{u}^{N, N+1}\left(x, \tau^{N}\right)$ is nonfinite), and $V_{u}^{N, N}\left(x, \tau^{N}\right) \leq V_{u}^{N, N+1}\left(x, \tau^{N}\right)+\alpha\left(\tau^{N}\right)$ if $V_{u}^{N, N+1}\left(x, \tau^{N}\right)$ is finite, (then $\beta\left(x, \tau^{N}\right)$ is finite). Thus, $\sup _{u \in U^{N, x, \tau}} V_{u}^{N, N+1}\left(x, \tau^{N}\right)=V^{N, N+1}\left(x, \tau^{N}\right) \leq \sup _{u \in U^{N, x, \tau}} V_{u}^{N, N}\left(x, \tau^{N}\right)+\alpha\left(\tau^{N}\right)=V^{N, N}\left(x, \tau^{N}\right)+\alpha\left(\tau^{N}\right)$, and, symmetrically, $V^{N, N}\left(x, \tau^{N}\right) \leq V^{N, N+1}\left(x, \tau^{N}\right)+\alpha\left(\tau^{N}\right)$ if $V^{N, N+1}\left(x, \tau^{N}\right)$ is finite $\left(\left(x, \tau^{N}\right) \in B^{N}\right)$.

The next to last inequality also holds if $U^{N, x, \tau}$ is empty. Define $\alpha\left(\tau^{N-1}\right):=E\left[\alpha\left(\tau^{N}\right) \mid \tau^{N-1}\right]$. The two last inequalities imply the two inequalities in what follows: for $\left(x, \tau^{N-1}\right) \in B^{N-1}$,

$$
\begin{aligned}
V_{u}^{N-1, N}\left(x, \tau^{N-1}\right)-\alpha\left(\tau^{N-1}\right)= & E\left[\int_{\hat{\tau}^{N-1}}^{\hat{\tau}^{N}} a f\left(s, x^{u}\left(s, \tau ; \tau_{N-1}, x\right), u(s), \tau\right) \mathrm{d} s\right. \\
& \left.+V^{N, N}\left(x^{u}\left(\hat{\tau}^{N}, \tau ; \tau_{N-1}, x\right), \tau^{N}\right)-\alpha\left(\tau^{N}\right) \mid \tau^{N-1}\right] \\
\leq & V_{u}^{N-1, N+1}\left(x, \tau^{N-1}\right) \\
= & E\left[\int_{\hat{\tau}^{N-1}}^{\hat{\tau}^{N}} a f\left(s, x^{u}\left(s, \tau ; \tau_{N-1}, x\right), u(s), \tau\right) \mathrm{d} s\right. \\
& \left.+V^{N, N+1}\left(x^{u}\left(\hat{\tau}^{N}, \tau ; \tau_{N-1}, x\right), \tau^{N}\right) \mid \tau^{N-1}\right] \\
\leq & E\left[\int_{\hat{\tau}^{N-1}}^{\hat{\tau}^{N}} a f\left(s, x^{u}\left(s, \tau ; \tau_{N-1}, x\right), u(s), \tau\right) \mathrm{d} s\right. \\
& \left.+V^{N, N}\left(x^{u}\left(\hat{\tau}^{N}, \tau ; \tau_{N-1}, x\right), \tau^{N}\right)+\alpha\left(\tau^{N}\right) \mid \tau^{N-1}\right] \\
= & V_{u}^{N-1, N}\left(x, \tau^{N-1}\right)+\alpha\left(\tau^{N-1}\right),
\end{aligned}
$$

so

$$
V_{u}^{N-1, N}\left(x, \tau^{N-1}\right)-\alpha\left(\tau^{N-1}\right) \leq V_{u}^{N-1, N+1}\left(x, \tau^{N-1}\right) \leq V_{u}^{N-1, N}\left(x, \tau^{N-1}\right)+\alpha\left(\tau^{N-1}\right)
$$

(the second inequality holds also if $V_{u}^{N-1, N+1}\left(x, \tau^{N-1}\right)$ is nonfinite, the first one holds if $V^{N-1, N+1}\left(x, \tau^{N-1}\right)$ is finite; then $V^{N, N+1}\left(x^{u}\left(\hat{\tau}^{N}, \tau ; \tau_{N-1}, x\right), \tau^{N}\right)$ is finite a.s. in $\left.\operatorname{Pr}\left[. \mid \tau^{N-1}\right]\right)$. Moreover, by the two last inequalities (in a shorthand notation)

$$
\begin{aligned}
V_{u}^{N-2, N}-\alpha & =E\left[a \int_{\hat{\tau}^{N-2}}^{\hat{\tau}^{N-1}}+V^{N-1, N}-\alpha\right] \leq V_{u}^{N-2, N+1} \\
& =E\left[a \int_{\hat{\tau}^{N-2}}^{\hat{\tau}^{N-1}}+V^{N-1, N+1}\right] \leq E\left[a \int_{\hat{\tau}^{N-2}}^{\hat{\tau}^{N-1}}+V^{N-1, N}+\alpha\right]=V_{u}^{N-2, N}+\alpha,
\end{aligned}
$$


and this continues backwards for $N-3, N-4, \ldots$, so for

$$
\alpha\left(\tau^{k}\right)=E\left[\alpha\left(\tau^{k+1}\right) \mid \tau^{k}\right]=E\left[E\left[\alpha\left(\tau^{k+2}\right) \mid \tau^{k+1}\right] \mid \tau^{k}\right]=E\left[\alpha\left(\tau^{k+2}\right) \mid \tau^{k}\right]=\ldots=E\left[\alpha\left(\tau^{N}\right) \mid \tau^{k}\right],
$$

for $\left(x, \tau^{k}\right) \in B^{k}, k \leq N$,

$$
V_{u}^{k, N}\left(x, \tau^{k}\right)-\alpha\left(\tau^{k}\right) \leq V_{u}^{k, N+1}\left(x, \tau^{k}\right) \leq V_{u}^{k, N}\left(x, \tau^{k}\right)+\alpha\left(\tau^{k}\right),
$$

(the first inequality holds if $V_{u}^{k, N+1}\left(x, \tau^{k}\right)$ is finite). Hence, for $k \leq N$,

$$
V^{k, N}\left(x, \tau^{k}\right)-\alpha\left(\tau^{k}\right) \leq V^{k, N+1}\left(x, \tau^{k}\right) \leq V^{k, N}\left(x, \tau^{k}\right)+\alpha\left(\tau^{k}\right),
$$

(the first inequality holds if $V^{k, N+1}\left(x, \tau^{k}\right)$ is finite).

Let $A:=\sup _{i} K_{i} / \bar{k}^{i}<\infty$. By $(2.5)$,

$$
E\left[\left[1_{[0, T]}\left(\tau_{N+1}\right) \mid \tau^{N}\right] \mid \tau^{k}\right] \leq \sum_{m=N+1}^{\infty} \operatorname{Pr}\left[T \in\left[\tau_{m}, \tau_{m+1}\right) \mid \tau^{k}\right] \leq \Phi^{*} k_{*}^{N+1-k} /\left(1-k_{*}\right) .
$$

Hence,

$$
\begin{aligned}
E\left[\alpha\left(\tau^{N}\right) \mid \tau^{k}\right] & =E\left[E\left[T\left(\hat{K}_{N}+\hat{K}_{N+1}\right) 1_{[0, T]}\left(\tau_{N+1}\right) \mid \tau^{N}\right] \mid \tau^{k}\right] \\
& \leq T\left(\hat{K}_{N}+\hat{K}_{N+1}\right) \Phi^{*} k_{*}^{N+1} / k_{*}^{k}\left(1-k_{*}\right) \\
& \leq \operatorname{AT}\left(\bar{k}^{N}+\bar{k}^{N+1}\right) \Phi^{*} k_{*}^{N+1} / k_{*}^{k}\left(1-k_{*}\right)=L_{k}\left(\bar{k} k_{*}\right)^{N+1},
\end{aligned}
$$

where $L_{k}:=A T(1 / \bar{k}+1) \Phi^{*} / k_{*}^{k}\left(1-k_{*}\right)$. By repeated use of $(2.21)$, for $\alpha_{N}^{k, N^{\prime}}=\sum_{M=N+1}^{N^{\prime}} L_{k}\left(\bar{k} k_{*}\right)^{M} \leq$ $L_{k}\left(\bar{k} k_{*}\right)^{N+1} /\left(1-\bar{k} k_{*}\right)$ and for $N^{\prime}>N$, we get the "iterated double inequality"

$$
V_{u}^{k, N}\left(x, \tau^{k}\right)-\alpha_{N}^{k, N^{\prime}} \leq V_{u}^{k, N^{\prime}}\left(x, \tau^{k}\right) \leq V_{u}^{k, N}\left(x, \tau^{k}\right)+\alpha_{N}^{k, N^{\prime}},\left(\left(x, \tau^{k}\right) \in B^{k}\right),
$$

(the first inequality holding if $V_{u}^{k, N^{\prime}}\left(x, \tau^{k}\right)$ is finite). Hence, for $\left(x, \tau^{k}\right) \in B^{k}$,

$$
V^{k, N}\left(x, \tau^{k}\right)-\alpha_{N}^{k, N^{\prime}} \leq V^{k, N^{\prime}}\left(x, \tau^{k}\right) \leq V^{k, N}\left(x, \tau^{k}\right)+\alpha_{N}^{k, N^{\prime}},
$$

(the first inequality holding if $V^{k, N^{\prime}}\left(x, \tau^{k}\right)$ is finite).

For later use, note that $\left|\sigma^{N+1}(\tau)\right| \leq \sum_{N \leq j<\infty}|a| \int_{\hat{\tau}_{j}}^{\hat{\gamma}_{j+1}} K_{j} \mathrm{~d} s$. Thus,

$$
\begin{aligned}
E\left[\left|\sigma^{N+1}(\tau)\right|\right] & \leq E\left[\sum_{j=N}^{\infty}|a| K_{j}\left(\hat{\tau}^{j+1}-\hat{\tau}^{j}\right)\right] \\
& \leq \sum_{j=N}^{\infty}|a| T K_{j} \operatorname{Pr}\left[\tau_{j}<T\right]=\sum_{j=N}^{\infty}|a| T K_{j} \sum_{m=j}^{\infty} \operatorname{Pr}\left[T \in\left(\tau_{m}, \tau_{m+1}\right]\right] \\
& \leq \sum_{j=N}^{\infty}|a| T K_{j} \sum_{m=j}^{\infty} \Phi^{*} k_{*}^{m} \leq \sum_{m=N}^{\infty}|a| T K_{j} \Phi^{*} k_{*}^{j} /\left(1-k_{*}\right) \\
& \leq \sum_{m=N}^{\infty} \Phi^{*} T|a| A\left(\bar{k} k_{*}\right)^{j} /\left(1-k_{*}\right) \leq \Phi^{*} T|a| A\left(\bar{k} k_{*}\right)^{N} /\left(1-\bar{k} k_{*}\right)\left(1-k_{*}\right) .
\end{aligned}
$$


Note that, by (2.23),

$$
W^{k, N+1}:=V^{k, N+1}-\sum_{j=0}^{N+1} L_{k}\left(\bar{k} k_{*}\right)^{j} \leq V^{k, N}-\sum_{j=0}^{N} L_{k}\left(\bar{k} k_{*}\right)^{j}=: W^{k, N},
$$

so the sequence $\left\{W^{k, N}\right\}_{N}$ is decreasing, hence $\lim _{N} W^{k, N}$ exists, and then also $\lim _{N} V^{k, N}=: V^{k, \infty}$ exists. In fact, for $\alpha_{N}^{k}=\lim _{N^{\prime} \rightarrow \infty} \alpha_{N}^{k, N^{\prime}}$, by (2.23),

$$
V^{k, k}\left(x, \tau^{k}\right)-\alpha_{k}^{k} \leq V^{k, \infty}\left(x, \tau^{k}\right) \leq V^{k, k}\left(x, \tau^{k}\right)+\alpha_{k}^{k},
$$

(the first inequality if $V^{k, \infty}\left(x, \tau^{k}\right)$ is finite).

Note that, by (2.16), and monotone convergence of $\left\{W^{k, N}\right\}_{N}$,

$$
V_{u}^{k, \infty}\left(x, \tau^{k}\right)=E_{\tau_{k+1}}\left[a \int_{\hat{\tau}^{k}}^{\hat{\tau}^{k+1}} f\left(s, x^{u}\left(s, \tau ; \tau_{k}, x\right), u(s), \tau\right) \mathrm{d} s+V^{k+1, \infty}\left(x^{u}\left(\hat{\tau}^{k+1}, \tau ; \tau_{k}, x\right), \tau^{k+1}\right) \mid \tau^{k}\right],
$$

so, for

$$
\begin{aligned}
\left(x, \tau^{k}\right) \in B^{k}, V^{k, \infty}\left(x, \tau^{k}\right)= & \sup _{u \in U^{k, x, \tau^{k}}} E_{\tau_{k+1}}\left[a \int_{\hat{\tau}^{k}}^{\hat{\tau}^{k+1}} f\left(s, x^{u}\left(s, \tau ; \tau_{k}, x\right), u(s), \tau\right) \mathrm{d} s\right. \\
& \left.+V^{k+1, \infty}\left(x^{u}\left(\hat{\tau}^{k+1}, \tau ; \tau_{k}, x\right), \tau^{k+1}\right) \mid \tau^{k}\right]
\end{aligned}
$$

Upper semicontinuity of $V^{k, \infty}$ Even $V^{k, \infty}$ is usc on $B^{k}$. To see this, let $\left(\bar{x}, \bar{\tau}^{k}\right) \in B^{k}$, and let $\left(x_{j}, \tau_{(j)}^{k}\right) \rightarrow\left(\bar{x}, \bar{\tau}^{k}\right)$, when $j \rightarrow \infty,\left(x_{j}, \tau_{(j)}^{k}\right) \in B^{k}$, the sequence being so chosen that $V^{k, \infty}\left(x_{j}, \tau_{(j)}^{k}\right) \rightarrow$ $\lim \sup _{(\check{x}, \tau) \in B^{k},\left(\check{x}, \tau^{k}\right) \rightarrow(\bar{x}, \bar{\tau})} V^{k, \infty}\left(\check{x}, \tau^{k}\right)$. If the last entity equals $-\infty$, there is nothing to prove. If not, $V^{k, \infty}\left(x_{j}, \tau_{(j)}^{k}\right)>-\infty$ for $j \geq$ some $j^{*}$. Then $V^{k, N^{\prime}}\left(x_{j}, \tau_{(j)}^{k}\right)>-\infty$ for $N^{\prime}$ large enough, in fact for all $N^{\prime} g e q k$, by (2.23) when $j \geq j^{*}$. Then, for any given $\varepsilon>0$, for $N^{*} \geq k$ chosen such that $\alpha_{N}^{k} \leq \varepsilon / 4$ for $N \geq N^{*}$, by (2.24), for any $j \geq j^{*}, V^{k, N}\left(x_{j}, \tau_{(j)}^{k}\right)-\varepsilon / 4 \leq V^{k, \infty}\left(x_{j}, \tau_{(j)}^{k}\right) \leq V^{k, N}\left(x_{j}, \tau_{(j)}^{k}\right)+\varepsilon / 4$. For some $j_{N} \geq j^{*}$, $V^{k, N}\left(x_{(j)}, \tau_{(j)}^{k}\right) \leq V^{k, N}\left(\bar{x}, \bar{\tau}^{k}\right)+\varepsilon / 2$ when $j \geq j_{N},\left(V^{k, N}\left(\check{x}, \tau^{k}\right)\right.$ is usc in $\left.\left(\check{x}, \tau^{k}\right) \in B_{j}^{k}\right)$. This means that for all $N \geq k, V^{k, N}\left(\bar{x}, \bar{\tau}^{k}\right)>-\infty$ and that $V^{k, N^{*}}\left(\bar{x}, \bar{\tau}^{k}\right) \leq V^{k, \infty}\left(\bar{x}, \bar{\tau}^{k}\right)+\varepsilon / 4$, by (2.24), so using (2.24) again, we get, for $j \geq j_{N^{*}}$, that

$V^{k, \infty}\left(x_{j}, \tau_{(j)}^{k}\right) \leq V^{k, N^{*}}\left(x_{j}, \tau_{(j)}^{k}\right)+\varepsilon / 4 \leq V^{k, N^{*}}\left(\bar{x}, \bar{\tau}^{k}\right)+\varepsilon / 4+\varepsilon / 2 \leq V^{k, \infty}\left(\bar{x}, \bar{\tau}^{k}\right)+\varepsilon / 4+\varepsilon / 2+\varepsilon / 4=V^{k, \infty}\left(\bar{x}, \bar{\tau}^{k}\right)+\varepsilon$.

Thus, $(\check{x}, \tau) \rightarrow V^{k, \infty}\left(\check{x}, \tau^{k}\right)$ is usc in $\left(\check{x}, \tau^{k}\right) \in B^{k}$.

Recall that $E\left[\left|\sigma^{N+1}(\tau)\right|\right] \rightarrow 0$, when $N \rightarrow \infty$. By (2.18) and the monotone convergence theorem (cf. the $W^{k, N}$ 's introduced above),

$$
E\left[a x^{u}(T, \tau)\right] \leq E\left[\sum_{0 \leq j \leq k-1} \int_{\hat{\tau}^{j}}^{\hat{\tau}^{j+1}} a f\left(s, x^{u}(s, \tau), u(s, \tau), \tau\right) \mathrm{d} s\right]+E\left[V^{k, \infty}\left(x^{u}\left(\hat{\tau}^{k}, \tau\right), \tau^{k}\right)\right] .
$$

Construction of optimal controls satisfying (2.26). Let us use (2.26) to define, by induction, measurable controls $u_{k}\left(t, \tau^{k}\right)$ that will turn out to give the optimal control: due to $(2.27)$ and the existence of an admissible solution, $V^{0, \infty}(0,0)$ is finite. Define $\left(u_{0}\left(t, \tau^{0}\right), x_{0}\left(t, \tau^{0}\right)\right)$ to be a control in $U^{0,0, \tau^{0}}$ with corresponding solution 
$x_{0}\left(t, \tau^{0}\right):=x_{0}\left(t, \tau^{0} ; 0,0\right)$ yielding supremum for $k=0$ in $(2.26)$, (such a control exists in $U^{0,0, \tau^{0}}$, by Rem. 2.3). Evidently, $\left(t, \tau^{0}\right) \rightarrow u_{0}\left(t, \tau^{0}\right)$ is measurable $\left(\tau^{0}=\tau_{0}=0\right.$, by Proposition $1, u_{0}\left(, \tau^{0}\right)$ is measurable in $\left.t\right)$. By induction, assume, for $j \leq k-1$ and for some measurable set $M_{j} \subset \Omega_{j}$ of full $P$-measure in $\Omega_{j}\left(\operatorname{Pr}\left[\Omega_{j} \backslash M_{j}\right]=\right.$ $0)$, that for each $\tau \in M_{j}$ a pair $\left(u_{j}\left(t, \tau^{j}\right), x_{j}\left(t, \tau^{j}\right)\right)$ exists such that $V^{j, \infty}\left(x_{j}\left(\tau_{j}, \tau^{j}\right), \tau^{j}\right)$ is finite, and such that the pair yields supremum in (2.26) for $k$ replaced by $j$, with $x=x_{j}\left(\tau_{j}, \tau^{j}\right),\left(x_{j}\left(\tau_{j}, \tau^{j}\right)=x_{j-1}\left(\tau_{j}, \tau^{j-1}\right)\right.$, $\left.x^{u}\left(., \tau ; \tau_{j}, x\right)=x_{j}\left(., \tau^{j}\right)\right)$, and with $u_{j}\left(., \tau^{j}\right) \in U^{j, x_{j-1}\left(\hat{\tau}_{j}, \tau^{j-1}\right), \tau},\left(t, \tau^{j}\right) \rightarrow u_{j}\left(t, \tau^{j}\right)$ measurable. By the induction hypothesis, $V^{k-1, \infty}\left(x_{k-1}\left(\hat{\tau}_{k-1}, \tau^{k-1}\right), \tau\right)$ is finite on $M_{k-1}$. Since $U^{k-1, x_{k-1}\left(\hat{\tau}_{k-1}, \tau^{k-1}\right), \tau}$ is nonempty for $\tau^{k-1} \in$ $M_{k-1}$ (it contains $u_{k-1}\left(., \tau^{k-1}\right)$ ), then, by $\left.(2.20)^{*}\right), V^{k-1, k-1}\left(x_{k-1}\left(\hat{\tau}_{k-1}, \tau^{k-1}\right), \tau^{k-1}\right)$ is bounded on $M_{k-1}$, and, by $(2.23), V^{k-1, N^{\prime}}\left(x_{k-1}\left(\hat{\tau}_{k-1}, \tau\right), \tau^{k-1}\right)$ is finite for $N^{\prime} \geq k-1$, so, by $(2.24), V^{k-1, \infty}\left(x_{k-1}\left(\tau_{k-1}, \tau^{k-1}\right), \tau^{k-1}\right)$ is a bounded function on $M_{k-1}$. Then, by (2.26),

$$
\begin{aligned}
1_{M_{k-1}} V^{k-1, \infty}\left(x_{k-1}\left(\hat{\tau}_{k-1,} \tau^{k-1}\right), \tau^{k-1}\right)= & 1_{M_{k-1}} E\left[a \int_{\hat{\tau}^{k-1}}^{\hat{\tau}^{k}} f\left(s, x_{k-1}\left(s, \tau^{k-1}\right), u_{k-1}\left(s, \tau^{k-1}\right), \tau\right) \mathrm{d} s\right. \\
& \left.\left.+V^{k, \infty}\left(x_{k-1}\left(\hat{\tau}^{k}, \tau^{k-1}\right), \tau^{k}\right)\right\} \mid \tau^{k-1}\right] .
\end{aligned}
$$

Taking expectation $\left(E\left[. \mid \tau^{0}\right]\right)$ on both sides yields a finite expression also on the right hand side. This means that $1_{M_{k-1}} V^{k, \infty}\left(x_{k-1}\left(\hat{\tau}^{k}, \tau^{k-1}\right), \tau^{k}\right)$ is a.s. finite, (otherwise $\left.E\left[E\left[1_{M_{k-1}} V^{k, \infty}\left(x_{k-1}\left(\hat{\tau}^{k}, \tau^{k-1}\right), \tau^{k}\right)\right\} \mid \tau^{k-1}\right]\right]$ would not be finite). I.e. a measurable subset $M^{k}$ of full $P$-measure in $\Omega_{k}$ exists such that

$V^{k, \infty}\left(x_{k-1}\left(\hat{\tau}^{k}, \tau^{k-1}\right), \tau^{k}\right)$ is finite for $\tau^{k} \in M^{k}$. Moreover, by Lusin's theorem, an increasing sequence of measurable sets $\left\{M_{k}^{j}\right\}_{j}$ exists, such that $\tau^{k} \mapsto V^{k, \infty}\left(x_{k-1}\left(\hat{\tau}^{k}, \tau^{k-1}\right), \tau^{k}\right)$ is continuous on $M_{k}^{j} \subset M^{k}$, with meas $\left(M^{k} \backslash M_{k}^{j}\right) \rightarrow 0$ when $j \rightarrow \infty$. For $\tau \in M_{k}^{j}$, by Remark 2.3 and (2.26) holding for $k$, a control $u_{k, \tau^{k}}(.) \in U^{k, x_{k-1}\left(\hat{\tau}_{k}, \tau^{k-1}\right), \tau}$, with corresponding solution $x_{k, \tau^{k}}($.$) satisfying x_{k, \tau^{k}}\left(\tau_{k}\right)=x_{k-1}\left(\tau_{k}, \tau^{k-1}\right)$ exists, yielding supremum in (2.26) for $x=x_{k, \tau^{k}}\left(\tau_{k}\right)$. Then the following equality is satisfied for $\tau \in M_{k}^{j}$ :

$$
V^{k, \infty}\left(x_{k-1}\left(\hat{\tau}^{k}, \tau^{k-1}\right), \tau^{k}\right)=E_{\tau_{k+1}}\left[a \int_{\hat{\tau}^{k}}^{\hat{\tau}^{k+1}} f\left(s, x_{k, \tau^{k}}(s), u_{k, \tau^{k}}(s), \tau\right) \mathrm{d} s+V^{k+1, \infty}\left(x_{k, \tau^{k}}\left(\hat{\tau}_{k+1}\right), \tau^{k+1}\right) \mid \tau^{k}\right]
$$

((2.29) reduces to $0=0$ when $\left.\tau_{k} \geq \hat{\tau}^{k}=T\right)$.

We want to choose $u_{k, \tau^{k}}($.$) to be simultaneously measurable in \left(t, \tau^{k}\right)$, in which case we write $u_{k}\left(t, \tau^{k}\right)$ instead of $u_{k, \tau^{k}}($.$) (and x_{k}\left(t, \tau^{k}\right)$ for the corresponding solution). For $\tau \in \hat{M}_{k}:=\cup_{j} M_{k}^{j}$, let $U_{\tau^{k}}^{k}$ be the set of controls in $U^{k, x_{k-1}\left(\hat{\tau}_{k}, \tau^{k-1}\right), \tau}$ for which (2.29) is satisfied. Define the functions $x^{k-1}\left(t, \tau^{k-1}\right), u^{k-1}\left(t, \tau^{k-1}\right)$ to be the nonanticipating functions that satisfy $\left(x^{k-1}\left(t, \tau^{k-1}\right), u^{k-1}\left(t, \tau^{k-1}\right)\right)=\left(x_{j}\left(t, \tau_{j}\right), u_{j}\left(t, \tau^{j}\right)\right)$ for $t \in\left(\tau_{j}, T\right]$, if $\tau \in M_{j}, j \leq k-1$, (then these functions are defined for P-a.e. $\tau$ in $\Omega_{k-1}$ ). Let $H_{j}^{k}, j=1,2, \ldots$ be measurable sets in $\Omega_{k}$ such that meas $\left(\Omega_{k} \backslash H_{j}^{k}\right)<1 / j$ and such that, by Lusin's theorem for Banach space valued measurable functions, $\tau \rightarrow u^{k-1}\left(., \tau^{k-1}\right): H_{j}^{k} \rightarrow L_{1}\left(J, \mathbb{R}^{r}\right)$ is continuous. Let $\tau_{(n)} \rightarrow \tau$, where $\tau, \tau_{(n)} \in F_{j}^{k}:=M_{k}^{j} \cap H_{j}^{k}$, and assume that $u_{k,\left(\tau_{(n)}\right)^{k}}(.) \rightarrow u($.$) in measure, u_{k,\left(\tau_{(n)}\right)^{k}}(.) \in U_{\left(\tau_{(n)}\right)^{k}}^{k}$. Then, using Ascoli's theorem, it is easily seen that a subsequence $x^{k-1}\left(t,\left(\tau_{\left(n_{j}\right)}\right)^{k-1}\right)$ is uniformly convergent to some continuous function $x($.$) on [0, T]$, which is a solution of $(2.2)$ on $[0, T]$ corresponding to $u^{k-1}\left(., \tau^{k-1}\right)$. Hence, by uniqueness, $x($.$) is equal to x^{k-1}\left(t, \tau^{k-1}\right)$. The subsequence is also chosen such that the solution $x_{k,\left(\tau_{\left(n_{j}\right)}\right)^{k}}(t)$ corresponding to $u_{k,\left(\tau_{(n)}\right)^{k}}($.$) , (which satisfies$ $x_{k,\left(\tau_{(n)}\right)^{k}}\left(\left(\tau_{(n)}\right)_{k}\right)=x^{k-1}\left(\left(\tau_{(n)}\right)_{k},\left(\tau_{(n)}\right)^{k-1}\right)$, converges for each $t$ in $\left(\tau_{k}, T\right]$ to some continuous function $x^{*}($. that is easily seen to be a solution of $(2.2)$ on $\left[\tau_{k}, T\right]$ with initial condition $x_{k, \tau^{k}}\left(\tau_{k}\right)=x^{k-1}\left(\tau_{k}, \tau^{k-1}\right)$. By uniqueness, $x^{*}(t)$ is equal to $x_{k, \tau^{k}}(t):=x^{u}\left(t, \tau ; x_{k-1}\left(\tau_{k}, \tau^{k-1}\right)\right)$. For $\tau^{k} \in F_{j}^{k}, x_{k, \tau^{k}}(T) \in B^{*}$ if $\tau_{k+1}>T$, provided $\operatorname{Pr}\left[\tau_{k+1}>T \mid \tau^{k}\right]>0$, since this inequality must hold for large $n_{j}$, by continuity in $\tau^{k}$. Furthermore, by continuity in $M_{k}^{j}$, for $\tau^{k}$ replaced by $\left(\tau_{\left(n_{j}\right)}\right)^{k}$ in (2.29), the left hand side converges to the left hand side 
as written in (2.29), when $n_{j} \rightarrow \infty$. For $\tau^{k}$ replaced by $\left(\tau_{\left(n_{j}\right)}\right)^{k}$ in the right hand side, by usc, the limsup of the right hand side when $n_{j} \rightarrow \infty$ is $\leq$ the right hand side as written, with $u_{k, \tau^{k}}()=.u($.$) . Hence, by (2.27)$, $u$ is optimal in $U^{k, x_{k-1}\left(\hat{\tau}_{k}, \tau^{k-1}\right), \tau}$, it belongs to $U_{\tau^{k}}^{k}$. Thus, when $\tilde{U}$ is furnished with the metric of convergence in measure, (in which it is separable and complete), the multifunction $\tau \rightarrow U_{\tau^{k}}^{k}$ is outer semi-continuous, (has the closed graph property), and hence is measurable, on each $F_{j}^{k}$, and therefore measurable on the set $M_{k}:=\cup_{j} F_{j}^{k}$ of full P-measure. By Kuratowski's measurable selection theorem, for each $\tau^{k} \in M_{k}$, a function $u_{k}\left(., \tau^{k}\right) \in U_{\tau^{k}}^{k}$ exists such that $\tau \rightarrow u_{k}\left(., \tau^{k}\right)$ is measurable on $M_{k}$. Then $(t, \tau) \rightarrow u_{k}\left(t, \tau^{k}\right)$ is measurable. Let $x_{k}(t, \tau)$ correspond to $u_{k}(t, \tau)$. Obviously, $\left(u_{k}\left(., \tau^{k}\right), x_{k}\left(., \tau^{k}\right)\right)$ is defined a.s. and yields supremum in (2.26) for $\left(x, \tau^{k}\right)=\left(x_{k-1}\left(\tau_{k}, \tau^{k-1}\right), \tau^{k}\right), \tau \in M_{k}$. As $x_{k-1}\left(\tau_{k}, \tau^{k-1}\right)=x_{k}\left(\tau_{k}, \tau^{k}\right)$ for $\tau_{k} \leq T, V^{k, \infty}\left(x\left(\tau_{k}, \tau^{k}\right), \tau^{k}\right)$ is finite on $M_{k}$.

Define $x^{*}(t, \tau), u^{*}(t, \tau)$ to be the nonanticipating functions that satisfy $\left(x^{*}(t, \tau), u^{*}(t, \tau)\right)=\left(x_{j}(t, \tau), u_{j}(t, \tau)\right)$ for $t \in\left(\tau_{j}, T\right]$ if $\tau \in M_{j}$. Then, a.s., $\left(x^{*}(t, \tau), u^{*}(t, \tau)\right)=\left(x_{j}(t, \tau), u_{j}(t, \tau)\right)$ if $t \in\left(\tau_{j}, \tau_{j+1}\right]$. Evidently, using (2.28) for $j=0,1, \ldots, k+1$, we get

$$
V^{0, \infty}(0,0)=\sum_{j=0}^{k} E\left[E\left[a \int_{\hat{\tau}^{j}}^{\hat{\tau}^{j+1}} f\left(s, x^{*}(s, \tau), u^{*}(s, \tau)\right) \mathrm{d} s \mid \tau^{j}\right] \mid \tau^{0}\right]+E\left[E\left[V^{k+1, \infty}\left(x_{k}\left(\hat{\tau}^{k+1}, \tau^{k}\right), \tau^{k+1}\right) \mid \tau^{k}\right] \mid \tau^{0}\right]
$$

By (2.24), the results $\lim E_{k \rightarrow \infty}\left[\alpha_{k}^{k} 1_{[0, T]}\left(\tau^{k}\right)\right]=0$ and $0 \leq E\left[E\left[T \hat{K}_{k+1} 1_{[0, T]}\left(\tau_{k+1}\right) \mid \tau^{k}\right] \mid \tau^{0}\right] \leq E\left[\alpha\left(\tau^{k}\right) \mid \tau^{0}\right]$ $\rightarrow 0$ when $k \rightarrow \infty$ (see comments subsequent to (2.24), and (2.20), for $N=k$, the last term (i.e. $\left.\left.E\left[V^{k+1, \infty}\left(x_{k}\left(\hat{\tau}^{k+1}, \tau^{k}\right), \tau^{k+1}\right) \mid \tau^{k}\right] \mid \tau^{0}\right]\right)$ goes to zero when $k \rightarrow \infty$, so letting $k \rightarrow \infty$ we get the following equality (for the convergence of the sum below, see the result $\lim _{N \rightarrow \infty} E\left[\mid \sigma_{N+1}\left(\tau^{N+1}\right)\right]=0$ obtained subsequent to $(2.24)$,

$$
V^{0, \infty}(0,0)=\sum_{j=0}^{\infty} E\left[a \int_{\hat{\tau}^{j}}^{\hat{\tau}^{j+1}} f\left(s, x^{*}(s, \tau), u^{*}(s, \tau)\right) \mathrm{d} s \mid \tau^{0}\right] .
$$

Hence, $u^{*}(.,$.$) is optimal. (Note that x^{*}(t, \tau)$ does satisfy $(2.3)$ and $(2.4)$, recall that $x_{k}\left(T, \tau^{k}\right) \in B^{*}$ when $\operatorname{Pr}\left[\tau_{k+1}>T \mid \tau^{k}\right]>0$, and notice that

$$
\begin{aligned}
\operatorname{Pr}\left[x^{*}(T, \tau) \in B^{*}\right] & =\sum_{k} \operatorname{Pr}\left[x^{*}(T, \tau) \in B^{*}, T \in\left[\tau_{k}, \tau_{k+1}\right)\right] \\
& =\sum_{k} \operatorname{Pr}\left[x_{k}\left(T, \tau^{k}\right) \in B^{*}, T \in\left[\tau_{k}, \tau_{k+1}\right)\right] \\
& =\sum_{k} \operatorname{Pr}\left[x_{k}\left(T, \tau^{k}\right) \in B^{*}, T<\tau_{k+1}, \tau_{k} \leq T\right] \\
& =\sum_{k} \operatorname{Pr}\left[x_{k}\left(T, \tau^{k}\right) \in B^{*} \mid T<\tau_{k+1}, \tau_{k} \leq T\right] \operatorname{Pr}\left[T<\tau_{k+1} \mid \tau_{k} \leq T\right] \operatorname{Pr}\left[\tau_{k} \leq T\right] \\
& =\sum_{k} \operatorname{Pr}\left[T<\tau_{k+1} \mid \tau_{k} \leq T\right] \operatorname{Pr}\left[\tau_{k} \leq T\right] \\
& \left.=\sum_{k} \operatorname{Pr}\left[T \in\left[\tau_{k}, \tau_{k+1}\right)\right]=1\right) .
\end{aligned}
$$

Remark 2.4. Below we need the following modifications of (2.9) and (2.10). There is given a closed set $A$ in $\mathbb{R}^{n}$ containing $x_{0}$, such that if $\bar{x} \in A \cap \operatorname{cl} B\left(x_{0}, r_{i-1}^{*}\left(\tau_{i}\right)\right)$, then $x^{u}\left(\tau_{i+1}, \tau ; \tau_{i}, \bar{x}\right) \in A \cap \operatorname{cl} B\left(x_{0}, r_{i}^{*}\left(\tau_{j}\right)\right), i=$ $0,1,2, \ldots, r_{-1}^{*}(t)=0$. For each $\tau$, for each $j \geq i \geq 1$, for $\bar{x} \in A \cap \operatorname{cl} B\left(x_{0}, r_{i-1}^{*}\left(\tau_{i}\right)\right)$, for some $\tau_{j}^{\prime} \in\left[\tau_{j}, \tau_{j+1}\right)$, the solution $x^{u}\left(t, \tau ; \tau_{i}, \bar{x}\right)$, belongs to $\operatorname{cl} B\left(x_{0}, \max \left\{n r_{j-1}^{*}(t), n r_{j}^{*}(t)\right\}\right)$ for $t \in\left(\tau_{j}, \tau_{j}^{\prime}\right] \cap J$ and to $\operatorname{cl} B\left(x_{0}, r_{j}^{*}(t)\right)$ for $t \in\left(\tau_{j}^{\prime}, \tau_{j+1}\right] \cap J$ (instead of to $\operatorname{cl} B\left(x_{0}, r_{j}^{*}(t)\right)$ for all $\left.t \in\left(\tau_{j}, \tau_{j+1}\right] \cap J\right)$. Furthermore, for each $\tau$, each $j \geq 0$, for some $\tau_{j}^{\prime} \in\left[\tau_{j}, \tau_{j+1}\right)$, the solution $x^{u}\left(t, \tau ; \tau_{0}, x_{0}\right)$ belongs to $\operatorname{cl} B\left(x_{0}, \max \left\{n r_{j-1}^{*}(t), n r_{j}^{*}(t)\right\}\right)$ for $t \in\left(\tau_{j}, \tau_{j}^{\prime}\right] \cap J$ and 
to $\operatorname{cl} B\left(x_{0}, r_{j}^{*}(t)\right)$ for $t \in\left(\tau_{j}^{\prime}, \tau_{j+1}\right] \cap J$ (instead of to $\operatorname{cl} B\left(x_{0}, r_{j}^{*}(t)\right)$ for all $\left.t \in\left(\tau_{j}, \tau_{j+1}\right] \cap J\right)$. Moreover, $(2.9)$ must be changed as follows: $\left|f_{0}(t, x, u, \tau)\right|,|f(t, x, u, \tau)| \leq K_{j}$ for $(x, u, \tau) \in \operatorname{cl} B\left(x_{0}, \max \left\{n r_{j-1}^{*}(t), n r_{j}^{*}(t)\right\}\right) \times U \times \Omega^{\prime \prime}$ when $t \in\left[\tau_{j}, \tau_{j}^{\prime}\right] \cap J$, and $\left|f_{0}(t, x, u, \tau)\right|,|f(t, x, u, \tau)| \leq K_{j}$ for $(x, u, \tau) \in \operatorname{cl} B\left(x_{0}, r_{j}^{*}(t)\right) \times U \times \Omega^{\prime \prime}$ when $t \in\left(\tau_{j}^{\prime}, \tau_{j+1}\right) \cap J$.

(Then the start points $x_{k-1, \tau^{k-1}}\left(\tau_{k}\right)$ belong to $A \cap \operatorname{cl} B\left(x_{0}, r_{k-1}^{*}\left(\tau_{k}\right)\right)$ and $f_{0}$ and $f$ are, as before, bounded by $K_{k}$ along the solutions $x_{k, \tau^{k}}(t)$ and $x^{u}\left(t, \tau ; \tau_{k}, \bar{x}\right), \bar{x} \in A \cap \operatorname{cl} B\left(x_{0}, r_{k-1}^{*}\left(\tau_{k}\right)\right)$, both properties being used in the proof).

Letting all $V^{k, N}\left(x, \tau^{k}\right)$, and $V^{k, \infty}\left(x, \tau^{k}\right)$ be defined only for $x \in A$, the proof will be a trivial modification of the proof above.

Finally, it is not necessary to assume uniqueness of solutions of (2.2) (or in (2.10)). It was done just to save a few words in the proof; uniqueness is not assumed in the crucial Proposition 2.2.

Remark 2.5. Let $Q$ be a closed set in $\mathbb{R}^{n+1}$. Theorem 2.2 holds even if the requirment $(t, x(t, \tau)) \in Q$ a.s. is added to the requirments in (2.6) for a pair to be admissible.

The proof has then to be changed as follows. In the arguments subsequent to (2.17) (and before (2.18)), the set $B^{k}$ has to be replaced by $B^{k} \cap\left\{\left(x, \tau^{k}\right):\left(\tau_{k}, x\right) \in Q\right\}$, and the points $(t, x)$ in the definitions of the two sets $S$ have to be restricted to the sets $\left\{(t, x):(t, x) \in Q\right.$ if $\left.\operatorname{Pr}\left[\tau_{N+1}>t \mid \tau^{N}\right]>0\right\}$ and $\{(t, x):(t, x)$ $\in Q$ if $\left.\operatorname{Pr}\left[\tau_{k}>t \mid \tau^{k-1}\right]>0\right\}$, respectively. Finally, the solution $x^{u}\left(t, \tau ; \tau_{k}, x\right)$ appearing in the definition of the set $U^{k, x, \tau}$, see the beginning of Continued proof of Theorem 2.2, has also to satisfy $\left(t, x^{u}\left(t, \tau ; \tau_{k}, x\right)\right) \in Q$ if $\left.\operatorname{Pr}\left[\tau_{k+1}>t \mid \tau^{k}\right]>0\right]$ a.s.

End conditions of the type $h^{k}(x(T, \tau))=0$ a.s, $k=1, \ldots, k^{\prime}$ and $h_{k}(x(T, \tau)) \geq 0$ a.s. $, k=1, \ldots, k^{\prime \prime}$, ( $h^{k}, h_{k}$ continuous), instead of (2.3) and (2.4), can also be allowed. In case of such end conditions, replacing $T$ by $T+1$, with $f$ and $f_{0}$ zero on $(T+1, T]$, and using auxiliary state variables $y^{k}$ and $y_{k}$ governed by $\dot{y}^{k}=h^{k}(x(t, \tau)) 1_{[T, T+1]}, \dot{y}_{k}=h_{k}(x(t, \tau)) 1_{[T, T+1]}$ reduce the problem to one with restrictions of the form $(2.3)$ and (2.4). (We can assume $h^{k}$ and $h_{k}$ to be bounded, if not, replace then by $h^{k} /\left(1+\left|h^{k}\right|\right)$ and $\left.h_{k} /\left(1+\left|h_{k}\right|\right)\right)$.

Remark 2.6. The weaker condition that $\mu\left(\tau_{j+1} \mid \tau_{0}, \ldots, \tau_{j}\right)$ is simply measurable in $\left(\tau_{j+1}, \tau_{0}, \ldots, \tau_{j}\right)$ suffices for Theorem 2.1 to hold. In this case, by Lusin's theorem, an increasing sequence of sets $\left\{\Omega_{m}^{i}\right\}_{m}$ exists, $\Omega_{m}^{i}$ relatively closed in $\Omega^{i}$, such that $\tau^{i} \rightarrow \mu\left(. \mid \tau^{i}\right) \in L_{1}(J, R)$ is continuous on each $\Omega_{m}^{i}$, with meas $\left[\Omega^{i} \backslash\left(\cup_{m} \Omega_{m}^{i}\right)\right]=0$. Similarly, the continuity condition on $f_{0}$ and $f$ can be weakened to continuity in $\left\{\left(t, x, u, \tau^{i}\right): t \in J, x \in \mathbb{R}^{n}, u \in U, \tau^{i} \in\right.$ $\left.\hat{\Omega}_{m}^{i}, \tau^{i} \leq t\right\}$, where $\left\{\hat{\Omega}_{m}^{i}\right\}_{m}$ is some increasing family of relatively closed sets for which meas $\left[\Omega^{i} \backslash\left(\cup_{m} \hat{\Omega}_{m}^{i}\right)\right]=0$ (a condition that comes not far from assuming mere measurability in $\tau^{i}$ ). Of cource, we may assume $\Omega_{m}^{i}=\hat{\Omega}_{m}^{i}$.

Some hints for a proof: by backwards induction, with $\Omega_{N, N+1}^{k}:=\Omega^{N+1}$, there exists an increasing sequence of sets $\left\{\Omega_{N, m}^{k}\right\}_{m}, k=N+1, N, \ldots, 1$, relatively closed in $\Omega^{k}$, such that $\operatorname{meas}\left(\Omega_{m}^{k} \backslash \Omega_{N, m}^{k}\right) \leq 2^{-N} / m\left(\Rightarrow 1_{\Omega_{N, m}^{k}} \uparrow\right.$ $1_{\Omega^{k}}$ a.e.), such that $\left\{\int_{\tau_{k}}^{\infty}\left(1_{\Omega^{k+1}}-1_{\Omega_{N, n}^{k+1}}\right) \mu\left(\tau_{k+1} \mid \tau^{k}\right) \mathrm{d} \tau_{k+1}\right\}_{n}$ converges uniformly in $\tau^{k}$ on each $\Omega_{N, m}^{k}$ to zero (use almost uniform convergence of $E\left[1_{\Omega^{k+1}}-1_{\Omega_{N, n}^{k+1}} \mid \tau_{k}\right]$ to zero). Define $\tilde{\Omega}_{m}^{k}=\cap_{N=1}^{\infty} \Omega_{N, m}^{k}$ and note that $1_{\tilde{\Omega}_{m}^{k}} \uparrow 1_{\Omega^{k}}$ a.e. Then it can be shown by backwards induction that $V^{k, N}$ is usc on $B^{k} \cap\left\{\mathbb{R}^{n} \times \Omega_{N, m}^{k}\right\}$ for each $m$, and hence that $V^{k, \infty}$ is usc on $\tilde{\Omega}_{m}^{k}$ for each $m$.

In fact, assuming that $V^{k, N}$ is usc on $B^{k} \cap\left\{\mathbb{R}^{n} \times \Omega_{N, m}^{k}\right\}$, the following property entails that $V^{k-1, N}$ is usc on $B^{k-1} \cap\left\{\mathbb{R}^{n} \times \Omega_{N, m}^{k-1}\right\}$ for each $m$ : assume that a sequence of triples $\left(\tau_{(j)}, x^{j}(),. u^{j}().\right)$ exists, such that $\tau_{(j)}^{k-1} \in \Omega_{N, m}^{k-1}, u^{j}(.) \in U^{k-1, x_{0}^{j}, \tau_{(j)}}, x^{j}($.$) a deterministic solution corresponding to u^{j}($.$) defined on I_{j}=\left[t_{0}^{j}, T\right]$, $t_{0}^{j}=\left(\tau_{(j)}\right)^{k-1}, x^{j}\left(t_{0}^{j}\right)=x_{0}^{j}$ and such that there exist $x_{0}, x^{*}(),. u^{*}(.) \in \tilde{U}, \tau$, with $\tau^{k-1} \in \Omega_{N, m}^{k-1}$, for which $\tau_{(j)}^{k-1} \rightarrow \tau^{k-1}, x_{0}^{j}=x^{j}\left(t_{0}^{j}\right) \rightarrow x_{0}, \sup _{t \in I_{j} \cap I}\left|x^{j}(t)-x^{*}(t)\right| \rightarrow 0$ for $I=\left[\tau_{k-1}, T\right], x^{*}\left(t_{0}\right)=x_{0}$ for $t_{0}=\tau_{k-1}$, with 
$\left(x^{*}(),. u^{*}().\right)$ satisfying $(2.12)$. Then ${ }^{6}$

$$
\begin{aligned}
\limsup _{j} E_{\tau_{k}}\left[a\left(x^{j}\left(\hat{\tau}^{k}\right)-x^{j}\left(t_{0}^{j}\right)\right)+\right. & \left.V^{k, N}\left(x^{j}\left(\hat{\tau}^{k}\right), \tau_{(j)}^{k-1}, \tau_{k}\right) \mid \tau_{(j)}^{k-1}\right] \leq E_{\tau_{k}}\left[a\left(x^{*}\left(\hat{\tau}^{k}\right)-x^{*}\left(t_{0}\right)\right)\right. \\
& \left.+V^{k, N}\left(x^{*}\left(\hat{\tau}^{k}\right), \tau^{k-1}, \tau_{k}\right) \mid \tau^{k-1}\right]
\end{aligned}
$$

\section{Piecewise continuous systems}

Let us now consider piecewise continuous systems, where the state jumps at the times $\tau_{i}$ introduced in Section 2 above. Hence, to the setup in Section 2, add the feature that

$$
x\left(\tau_{i}+, \tau\right)=\hat{g}\left(\tau_{i}, x\left(\tau_{i}-, \tau\right), i\right), i=1,2, \ldots
$$

So now, $t \rightarrow x(t, \tau)$ is only absolutely continuous (and governed by the differential equation in (2.2)) between the points $\tau_{i}$, with left and right limits at each $\tau_{i}, i=1,2, \ldots$ satisfying $(3.1)$. We take $t \rightarrow x(t, \tau)$ to be left continuous. The functions $f_{0}$ and $f$ satisfy the general assumptions as before, and $\hat{g}$ is continuous. It is assumed that, for some constants $\alpha, \kappa, \alpha_{g}$, and $\kappa_{g}$, for all $(t, x, u, \tau) \in J \times \mathbb{R}^{n} \times U \times \Omega^{\prime \prime},|f(t, x, u, \tau)| \leq \alpha+\kappa|x|$, $\left|f_{0}(t, x, u, \tau)\right| \leq \alpha+\kappa|x|$, and $|\hat{g}(t, x, i)| \leq \alpha_{g}+\kappa_{g}|x|$ (for all $i$ ). For $h^{*} \equiv 0$, we now want to maximize the criterion in (2.1).

Theorem 3.1. Assume that the components $g^{m}$ of $g:=\hat{g}-x$ satisfy $g^{m} \equiv 0$ for $m=1, \ldots, n_{1}$, and $g^{m} \geq 0$ for $m=n_{1}+1, \ldots, m_{2}$. Assume also that $k_{*}$ in (2.5) satisfies $k_{*}<1 / \kappa_{g}$. Assume, finally, that an admissible solution $(x(t, \tau), u(t, \tau))$ of $(2.2),(3.1)$ exists, that $U$ is compact, and that $N(t, x, \tau)$ is convex (see (8)). Then there exists an optimal pair $\left(x^{*}(t, \tau), u^{*}(t, \tau)\right)$.

Remark 3.2. If the assumptions on the components $g^{m}, m=1, \ldots, n_{2}$ fail, then we run the risk that no admissible solution exists. (See the discussion in Sect. 3.4 in Seierstad [10]). Formally the conditions $g^{m} \equiv 0$ for $m=1, \ldots, n_{1}$, and $g^{m} \geq 0$ for $m=n_{1}+1, \ldots, m_{2}$ can be dropped.

It is not difficult to carry out essentially the same proof as above even in the present jump situation, it would add some few more details. However, being more than long enough, we did not want the proof to become even longer by adding in these extra details. So, instead we shall use Theorem 2.1 in an suitably rewritten system to obtain Theorem 3.1, even if that necessitates some tedious, mainly "book-keeping" arguments.

Proof. Theorem 2.1 holds for any norm $|x|$ on $\mathbb{R}^{n}$ equivalent to the Euclidean norm, and given this norm, we shall use the max-norm $|(z, y)|=\max \{|z|,|y|\}$ on $\mathbb{R}^{n} \times \mathbb{R}^{n}$. Define $\bar{x}=\left(\bar{x}^{1}, \ldots, \bar{x}^{n_{2}}, 0, \ldots, 0\right) \in \mathbb{R}^{n}$ and $\lambda(t)=x_{0}+(t / T)\left(\bar{x}-x_{0}\right)$. Let us introduce translated trajectories $\check{x}(t, \tau):=x(t, \tau)-\lambda(t)$, governed by the system

$$
\begin{aligned}
\mathrm{d} \check{x} / \mathrm{d} t & =\check{f}(t, \check{x}, u, \tau):=f(t, \check{x}+\lambda(t), u, \tau)-(1 / T)\left(\bar{x}-x_{0}\right), \check{x}(0)=0, \\
\check{x}\left(\tau_{i}+, \tau\right) & =\check{g}\left(\tau_{i}, \check{x}\left(\tau_{i}-, \tau\right), i\right):=\hat{g}\left(\tau_{i}, \check{x}\left(\tau_{i}-, \tau\right)+\lambda\left(\tau_{i}\right), i\right)-\lambda\left(\tau_{i}\right),
\end{aligned}
$$

\footnotetext{
${ }^{6}$ To prove this inequality, note that $-K_{*} \leq a\left(x^{j}\left(\hat{\tau}^{k}\right)-x^{j}\left(t_{0}^{j}\right)\right)+V^{k, N}\left(x^{j}\left(\hat{\tau}^{k}\right), \tau^{k}\right) \leq K_{*}$ for some positive $K_{*}$ independent of $\tau^{k}$ and $j$, (the left hand inequality holding only if $V^{k, N}\left(x^{j}\left(\hat{\tau}^{k}\right), \tau^{k}\right)$ is $\left.>-\infty\right)$, and, hence, for $n$ large, $\alpha^{j}:=E_{\tau_{k}}\left[\left\{a\left(x^{j}\left(\hat{\tau}^{k}\right)-x^{j}\left(t_{0}^{j}\right)\right)+\right.\right.$ $\left.\left.V^{k, N}\left(x^{j}\left(\hat{\tau}^{k}\right), \tau_{(j)}^{k-1}, \tau_{k}\right)\right\} 1_{\Omega^{k}}\left(\tau_{(j)}^{k-1}, \tau_{k}\right) \mid \tau_{(j)}^{k-1}\right] \leq E_{\tau_{k}}\left[\left\{a\left(x^{j}\left(\hat{\tau}^{k}\right)-x^{j}\left(t_{0}^{j}\right)\right)+V^{k, N}\left(x^{j}\left(\hat{\tau}^{k}\right), \tau_{(j)}^{k-1}, \tau_{k}\right)\right\} 1_{\Omega_{N, n}^{k}}\left(\tau_{(j)}^{k-1}, \tau_{k}\right) \mid \tau^{k-1}\right]+\varepsilon$, because for $n$ large $E_{\tau_{k}}\left[\left\{a\left(x\left(\hat{\tau}^{k}\right)-x\left(t_{0}^{j}\right)\right)+V^{k, N}\left(x^{j}\left(\hat{\tau}^{k}\right), \tau_{(j)}^{k-1}, \tau_{k}\right)\right\} 1_{\Omega^{k} \backslash \Omega_{N, n}^{k}} \mid \tau_{(j)}^{k-1}\right] \leq \varepsilon$ uniformly in $j$. For some subsequence $j_{i}$ both $\lim \alpha^{j_{i}}=\lim \sup _{j} \alpha^{j}$ and $\mu\left(. \mid \tau_{\left(j_{i}\right)}^{k-1}\right) \rightarrow \mu\left(. \mid \tau^{k-1}\right)$ a.s. Then, by Fatou's lemma, $\lim _{j_{i}} \alpha^{j}=\varepsilon+\limsup _{j_{i}} E_{\tau_{k}}\left[\left\{a\left(x^{j_{i}}\left(\hat{\tau}^{k}\right)-x\left(t_{0}^{j_{i}}\right)\right)+\right.\right.$ $\left.\left.V^{k, N}\left(x^{j_{i}}\left(\hat{\tau}^{k}\right), \tau_{\left(j_{i}\right)}^{k-1}, \tau_{k}\right)\right\} 1_{\Omega_{N, n}^{k}}\left(\tau_{\left(j_{i}\right)}^{k-1}, \tau_{k}\right) \mid \tau_{\left(j_{i}\right)}^{k-1}\right] \leq \varepsilon+E_{\tau_{k}}\left[\left\{a\left(x^{*}\left(\hat{\tau}^{k}\right)-x^{*}\left(t_{0}\right)\right)+V^{k, N}\left(x^{*}\left(\hat{\tau}^{k}\right), \tau^{k-1}, \tau_{k}\right)\right\} 1_{\Omega_{N, n}^{k}}\left(\tau^{k-1}, \tau_{k}\right) \mid \tau^{k-1}\right]$. Letting $n \rightarrow \infty$, then $\varepsilon \rightarrow 0$ and we get the asserted inequality. (From this we also get that solutions $u_{k, \tau_{k}}(t)$ in the section subsequent to (2.27) again exist).
} 
with criterion integrand $\check{f}_{0}(t, \check{x}, u, \tau):=f_{0}(t, \check{x}+\lambda(t), u, \tau)$. Note that if $\lambda^{*}=\max _{t \in J}|\lambda(t)|$, then $|\check{f}(t, \check{x}, u, \tau)| \leq$ $\check{\alpha}+\kappa|\check{x}|,\left|\check{f}_{0}(t, \check{x}, u, \tau)\right| \leq \check{\alpha}+\kappa|\check{x}|,\left|\check{g}\left(\tau_{i}, \check{x}\left(\tau_{i}-, \tau\right), i\right)\right| \leq \check{\alpha}_{g}+\kappa_{g}|\check{x}|, \check{\alpha}=(1 / T)\left|x_{0}-\bar{x}\right|+\alpha+\kappa \lambda^{*}, \check{\alpha}_{g}=\lambda^{*}+\alpha_{g}+\kappa_{g} \lambda^{*}$. The end condition on $\breve{x}(T, \tau)$ is $\check{x}^{m}(T, \tau)=0$ a.s. for $m=1, \ldots, n_{1}, \breve{x}^{m}(T, \tau) \geq 0$ a.s. for $m=n_{1}+1, \ldots, n_{2}$. Below, we write $x, x_{0}, f, f_{0, g}, \alpha$, and $\alpha_{g}$ instead of $\check{x}, \check{x}_{0}, \check{f}, \check{f}_{0}, \check{g}, \check{\alpha}$, and $\check{\alpha}_{g}$.

A. Assume first that there exist two sequences of positive numbers $M_{i}, K_{i}$, and positive continuous nondecreasing functions $r_{i}^{*}(),. i=0,1, \ldots, \sup _{i, t \in[0, T]} r_{i}^{*}(t) / \bar{k}^{i}<\infty$ and $\sup _{i} K_{i} / \bar{k}^{i}<\infty$ for some $\bar{k} \in\left(1,1 / k_{*}\right)$, and $\sum \sqrt{M_{i}}<\infty$, such that $|f(t, x, u, \tau)| \leq K_{i}$ and $\left|f_{0}(t, x, u, \tau)\right| \leq K_{i}$ for all $(x, u, \tau) \in \operatorname{cl} B\left(0, r_{i}^{*}(t)\right) \times U \times \Omega^{\prime \prime}$, for all $t \in\left(\tau_{i}, \tau_{i+1}\right]$, and such that, for any control $u(.,.) \in U^{\prime}$ and any $\tau \in \Omega^{\prime \prime}$, with $\tau_{k} \in(0, T)$, and any $\tilde{x} \in \operatorname{cl} B\left(0, r_{k-1}^{*}\left(\tau_{k}\right)\right)$, any solution $x\left(t, \tau ; \tau_{k}, \tilde{x}\right), t \in\left[\tau_{k}, T\right]$, of $\dot{x}=f(t, x, u(t), \tau)$ starting at $\left(\tau_{k}, \tilde{x}\right)$ (i.e. $x\left(\tau_{k}-, \tau ; \tau_{k}, \tilde{x}\right)=\tilde{x}$ ) and satisfying the jump condition (3.1) for $i \geq k$, by assumption satisfies $x\left(t, \tau ; \tau_{k}, \tilde{x}\right) \in$ $\operatorname{cl} B\left(0, r_{j}^{*}(t)\right)$ for $t \in\left(\tau_{j}, \tau_{j+1}\right] \cap J, j \geq k$. Moreover, $x(t, \tau ; 0,0) \in \operatorname{cl} B\left(0, r_{j}^{*}(t)\right)$ for $t \in\left(\tau_{j}, \tau_{j+1}\right) \cap J, j=0,1, \ldots$, for any solution $x(t, \tau ; 0,0)$ on $[0, T]$. Assume moreover that $|\hat{g}(t, x, i)-x| \leq M_{i}$ when $|x| \leq \max \left\{n r_{i-1}^{*}(t), n r_{i}^{*}(t)\right\}$. (These conditions are called the auxiliary conditions). This jumping system can be rewritten as a nonjumping system as follow:

let $M=\sum_{i=1}^{\infty}\left(M_{i}+\sqrt{M_{i}}\right), M_{0}=0, a_{j}:=\sum_{i=0}^{j}\left(M_{i}+\sqrt{M_{i}}\right)$, and $b_{j}:=M_{j}+\sqrt{M_{j}}$. For $i=1,2, \ldots$, let $\sigma_{i}:=\sigma_{i}\left(\tau_{i}\right):=\tau_{i}+a_{i-1}$ if $\tau_{i}<T$, and $\sigma_{i}:=\sigma_{i}\left(\tau_{i}\right):=T+M+\tau_{i}$ if $\tau_{i} \geq T$, moreover, let $\sigma_{0}=0$. There is an one-one correspondence between the $\sigma_{i}$ 's and the $\tau_{i}$ 's. Note that $\sigma_{i}<T+M \Leftrightarrow \tau_{i}<T$. In an obvious way, the densities $\mu\left(\tau_{k} \mid \tau^{k-1}\right)$ give rise to densities $\mu^{*}\left(\sigma_{k} \mid \sigma^{k-1}\right), k=0,1,2 \ldots$, that, by the way, are equal to zero on $\left[\sigma_{k-1}, \sigma_{k-1}+b_{k-1}\right] \cap[0, T+M]$.

Let $\sigma=\left(\sigma_{0}, \sigma_{1}, \ldots\right)$ and let $v\left(t, \sigma_{0}, \sigma_{1}, \ldots\right)$ take values in $U$, be nonanticipating and simultaneously measurable on each set $[0, T+M] \times \Omega_{i}^{\prime}, \Omega_{i}^{\prime}:=\left\{\left(\sigma_{0}, \sigma_{1}, \ldots\right): \sigma_{i+1}>T+M\right\}$. (The set of such controls is denoted $\left.U^{\prime \prime}\right)$. For $t \in[0, T+M]$, define

$$
\begin{gathered}
h_{0}\left(t, z(.), v, \sigma_{0}, \sigma_{1}, \ldots\right)=\sum_{i=0}^{\infty} f_{0}\left(t-a_{i}, z(t), v, \tau_{0}, \tau_{1}, \ldots\right) 1_{\left(\sigma_{i}+b_{i}, \sigma_{i+1}\right]}(t) \text { and for } g:=\hat{g}-x, \\
h\left(t, z(.), v, \sigma_{0}, \sigma_{1}, \ldots\right)=\sum_{i=0}^{\infty} f\left(t-a_{i}, z(t), v, \tau_{0}, \tau_{1}, \ldots\right) 1_{\left(\sigma_{i}+b_{i}, \sigma_{i+1}\right]}(t)+\sum_{i=1}^{\infty} g\left(\tau_{i}, z\left(\sigma_{i}\right), i\right) 1_{\left(\sigma_{i}, \sigma_{i}+M_{i}\right]}(t) / M_{i} .
\end{gathered}
$$

Then, for any given $v(t, \sigma)$, let $z^{v}(t, \sigma):=z(t, \sigma)$, for $t \in[0, T+M]$, be the solution - continuous in $t$ - of the retarded equation

$$
\dot{z}(t, \sigma)=h(t, z(.), v(t, \sigma), \sigma), z(0, \sigma)=0 .
$$

Define, for $s \in[0, T]$,

$$
x(s, \tau)=\sum_{i=0}^{\infty} z\left(s+a_{i}, \sigma\right) 1_{\left(\sigma_{i}+b_{i}, \sigma_{i+1}\right]}\left(s+a_{i}\right),
$$

and

$$
u(s, \tau)=\sum_{i=0}^{\infty} v\left(s+a_{i}, \sigma\right) 1_{\left(\sigma_{i}+b_{i}, \sigma_{i+1}\right]}\left(s+a_{i}\right) .
$$


Now, $z(t, \sigma)$ satisfies $\dot{z}(t, \sigma)=f\left(t-a_{i}, z(t, \sigma), v(t, \sigma), \tau_{0}, \tau_{1}, \ldots\right)$ for $t \in\left(\sigma_{i}+b_{i}, \sigma_{i+1}\right], t \leq T+M$. Assume $\tau_{i}<T$. Then, for $t^{\prime} \in\left[\tau_{i}, \tau_{i+1}\right), t^{\prime} \leq T$,

$$
\begin{aligned}
x\left(t^{\prime}, \tau\right)-x\left(\tau_{i}+, \tau\right) & =z\left(t^{\prime}+a_{i}, \sigma\right)-z\left(\sigma_{i}+b_{i}, \sigma\right) \\
& =\int_{\sigma_{i}+b_{i}}^{t^{\prime}+a_{i}} f\left(t-a_{i}, z(t, \sigma), v(t, \sigma), \tau\right) \mathrm{d} t \\
& =\int_{\tau_{i}}^{t^{\prime}} f\left(s, z\left(s+a_{i}, \sigma\right), v\left(s+a_{i}, \sigma\right), \tau\right) \mathrm{d} s \\
& =\int_{\tau_{i}}^{t^{\prime}} f(s, x(s, \tau), u(s, \tau), \tau) \mathrm{d} s .
\end{aligned}
$$

Note that $z(t, \sigma)$ is constant on $\left(\sigma_{i}+M_{i}, \sigma_{i}+b_{i}\right)$. Moreover, for $\tau_{i}<T$,

$$
\begin{aligned}
x\left(\tau_{i}+, \tau\right)-x\left(\tau_{i}-, \tau\right) & =z\left(\sigma_{i}+M_{i}, \sigma\right)-z\left(\sigma_{i}, \sigma\right) \\
& =\int_{\sigma_{i}}^{\sigma_{i}+M_{i}}\left(1 / M_{i}\right) g\left(\tau_{i}, z\left(\sigma_{i}, \sigma\right), i\right) \mathrm{d} t \\
& =g\left(\tau_{i}, z\left(\sigma_{i}, \sigma\right), i\right)=g\left(\tau_{i}, x\left(\tau_{i}-, \tau\right), i\right) .
\end{aligned}
$$

Hence, $(x(., \tau), u(., \tau))$ satisfies $(2.2)$ and (3.1). Symmetrically, if $(x(),. u()$.$) satisfies (2.2)$ and (3.1), there is a pair $(z(.,),. v(.,)$.$) satisfying (3.2),(u(., \tau)$ and $v(., \sigma)$ again related as in (3.4)).

Now, (3.2) is a retarded differential equation. There would be no problem if Theorem 2.1 was proved for nonjumping states governed by retarded equations, (and the proof would be almost the same). But let us stick to ordinary equations: we shall work with two states, $z$, developing as before, and $y$, being equal to $z$, except on each $\left(\sigma_{i}, \sigma_{i}+M_{i}\right]$, where it is constant and equals $z\left(\sigma_{i}, \sigma\right)$, and on each $\left(\sigma_{i}+M_{i}, \sigma_{i}+b_{i}\right]$ where it develops in such a manner that it reaches the constant value $z$ has on $\left(\sigma_{i}+M_{i}, \sigma_{i}+b_{i}\right]$ before the end of the interval, (in particular, $y\left(\sigma_{i}+b_{i}, \sigma\right)=z\left(\sigma_{i}+b_{i}, \sigma\right)$ ).

Define

$$
h_{1}\left(t, z, y, v, \sigma_{0}, \sigma_{2}, \ldots\right)=\sum_{i=0}^{\infty} f\left(t-a_{i}, z, v, \tau_{0}, \tau_{1}, \ldots\right) 1_{\left(\sigma_{i}+b_{i}, \sigma_{i+1}\right]}(t)+\sum_{i=1}^{\infty} g\left(\tau_{i}, y, i\right) 1_{\left(\sigma_{i}, \sigma_{i}+M_{i}\right]}(t) / M_{i},
$$

and

$$
h_{2}\left(t, z, y, v, \sigma_{0}, \sigma_{1}, \ldots\right)=\sum_{i=0}^{\infty} f\left(t-a_{i}, z, v, \tau_{0}, \tau_{1}, \ldots\right) 1_{\left(\sigma_{i}+b_{i}, \sigma_{i+1}\right]}(t)+\sum_{i=1}^{\infty} H(z, y) 1_{\left(\sigma_{i}+M_{i}, \sigma_{i}+b_{i}\right]}(t),
$$

where $H(z, y)$ has the components $H^{m}:=H^{m}\left(z^{m}, y^{m}\right):=-2\left(y^{m}-z^{m}\right)^{1 / 2}$ if $y^{m} \geq z^{m}, H^{m}:=2\left(z^{m}-y^{m}\right)^{1 / 2}$ if $y^{m}<z^{m}, m=1, \ldots, n$. Evidently, $H$ is continuous. The equations governing $z$ and $y$ are $\dot{z}=h_{1}(t, z, y, v, \sigma)$ and $\dot{y}=h_{2}(t, z, y, v, \sigma), z(0)=y(0)=0$. Define $\gamma_{i}=z^{m}\left(\sigma_{i}\right)-z^{m}\left(\sigma_{i}+M_{i}\right)$ and note that

$$
\left|\gamma_{i}\right|=\left|z^{m}\left(\sigma_{i}\right)-z^{m}\left(\sigma_{i}+M_{i}\right)\right| \leq\left|\int_{\sigma_{i}}^{\sigma_{i}+M_{i}}\left(1 / M_{i}\right) g^{m}\left(\tau_{i}, z\left(\sigma_{i}, \sigma\right), i\right) \mathrm{d} t\right| \leq \int_{\sigma_{i}}^{\sigma_{i}+M_{i}} 1 \mathrm{~d} t=M_{i},
$$

when $\sigma_{i}<T+M$. Now, the equation $\dot{y}^{m}=H^{m}\left(z^{m}, y^{m}\right), y^{m}\left(\sigma_{i}+M_{i}\right)=z^{m}\left(\sigma_{i}\right)$ given, has the unique solution

$$
y^{m}(t)=\left(-t+\sigma_{i}+M_{i}+\sqrt{\gamma_{i}}\right)^{2}+z^{m}\left(\sigma_{i}+M_{i}\right)
$$

on $\left(\sigma_{i}+M_{i}, \sigma_{i}+M_{i}+\sqrt{\gamma_{i}}\right] \subset\left(\sigma_{i}+M_{i}, \sigma_{i}+M_{i}+\sqrt{M_{i}}\right]$ if $\gamma_{i} \geq 0$, and if $\gamma_{i}<0$, then

$$
y^{m}(t)=-\left(-t+\sigma_{i}+M_{i}+\sqrt{-\gamma_{i}}\right)^{2}+z^{m}\left(\sigma_{i}+M_{i}\right)
$$


on $\left(\sigma_{i}+M_{i}, \sigma_{i}+M_{i}+\sqrt{-\gamma_{i}}\right]$, whereas $y^{m}(t)=z^{m}\left(\sigma_{i}+M_{i}\right)$ on $\left(\sigma_{i}+M_{i}+\sqrt{\left|\gamma_{i}\right|}, \sigma_{i}+b_{i}\right]$, recall that $z(t)$ is constant on $\left(\sigma_{i}+M_{i}, \sigma_{i}+b_{i}\right]$. Define the continuous function $r_{i}^{* *}(t)$ by $r_{i}^{* *}(t)=r_{i}^{*}\left(t-a_{i-1}\right)$ for $t \in\left[a_{i-1}, T+a_{i-1}\right]$, with $r_{i}^{* *}(t)$ constant on $\left[0, a_{i}\right]$ and on $\left[T+a_{i}, T+M\right]$. When $t \in\left[\sigma_{j}+b_{j}, \sigma_{j+1}\right), t<T+M$ and $(\bar{z}, \bar{y})=(\bar{x}, \bar{x}) \in \operatorname{cl} B\left((0,0), r_{i-1}^{* *}\left(\sigma_{i}\right)\right)$ (so $\left.\bar{x} \in \operatorname{cl} B\left(0, r_{i-1}^{*}\left(\tau_{i}\right)\right)\right)$, then $\left(z\left(t, \sigma ; \sigma_{i},(\bar{z}, \bar{y})\right), y\left(t, \sigma ; \sigma_{i},(\bar{z}, \bar{y})\right)\right)$ and $(z(t, \sigma ; 0,(0,0)), y(t, \sigma ; 0,(0,0)))$ belong to $\operatorname{cl} B\left((0,0), r_{j}^{* *}(t)\right)$, where

$$
\begin{aligned}
z\left(t, \sigma ; \sigma_{i},(\bar{z}, \bar{y})\right) & =y\left(t, \sigma ; \sigma_{i},(\bar{z}, \bar{y})\right) \\
& =x\left(t-a_{j}, \tau ; \tau_{i}, \bar{x}\right) \in \operatorname{cl} B\left(0, r_{j}^{*}\left(t-a_{i}\right)\right) \subset \operatorname{cl} B\left(0, r_{j}^{*}\left(t-a_{i-1}\right)\right)=\operatorname{cl} B\left(0, r_{j}^{* *}(t)\right) .
\end{aligned}
$$

Moreover, when $t \in\left[\sigma_{j}, \sigma_{j}+b_{j}\right), t<T+M$, then the components $z^{m}\left(t, \sigma ; \sigma_{i},(\bar{z}, \bar{y})\right)$ and $y^{m}\left(t, \sigma ; \sigma_{i},(\bar{z}, \bar{y})\right)$ belong to

$$
\begin{aligned}
{\left[x^{m}\left(\tau_{j}-, \tau ; \tau_{i}, \bar{x}\right), x^{m}\left(\tau_{j}+, \tau ; \tau_{i}, \bar{x}\right)\right] } & \subset \operatorname{cl} B\left(0, \max \left\{r_{j-1}^{*}\left(\tau_{j}\right), r_{j}^{*}\left(\tau_{j}\right)\right\}\right) \\
& =\operatorname{cl} B\left(0, \max \left\{r_{j-1}^{* *}\left(\tau_{j}+a_{j-2}\right), r_{j}^{* *}\left(\tau_{j}+a_{i-1}\right)\right\}\right) \\
& \subset \operatorname{cl} B\left(0, \max \left\{r_{j-1}^{* *}\left(\tau_{j}+a_{j-1}\right), r_{j}^{* *}\left(\tau_{j}+a_{j-1}\right)\right\}\right) \\
& =\operatorname{cl} B\left(0, \max \left\{r_{i-1}^{* *}\left(\sigma_{j}\right), r_{i}^{* *}\left(\sigma_{j}\right)\right\}\right),
\end{aligned}
$$

so $\left(z\left(t, \sigma ; \sigma_{i},(\bar{z}, \bar{y})\right), y\left(t, \sigma ; \sigma_{i},(\bar{z}, \bar{y})\right)\right) \in \operatorname{cl} B\left((0,0), \max \left\{n r_{j-1}^{* *}(t), n r_{j}^{* *}(t)\right\}\right)$. Similarly, when $t \in\left[\sigma_{j}, \sigma_{j+1}+b_{j}\right)$, $t<T+M,(z(t, \sigma ; 0,(0,0)), y(t, \sigma ; 0,(0,0))) \in \operatorname{cl} B\left((0,0), \max \left\{n r_{j-1}^{* *}(t), n r_{j}^{*}(t)\right\}\right)$. Define $r_{i}^{\prime \prime}:=\max _{t} r_{i}^{* *}(t)$. Due to the auxiliary conditions, this system $\left(i . e .\left(h_{0}, h_{1}, h_{2}\right)\right)$ satisfies all conditions placed upon a nonjumping system in Theorem 2.1, combined with Remark 4 above: the property $\left|h_{0}(t, z, u, \sigma)\right|,\left|h_{1}(t, z, y, u, \sigma)\right|,\left|h_{2}(t, z, y, u, \sigma)\right| \leq$ $\tilde{K}_{i}:=\max \left\{n, 4 n^{2} r_{i-1}^{\prime \prime 2} r_{i}^{\prime \prime}, K_{i}\right\}$ holds for $t \in\left(\sigma_{i}, \sigma_{i}+b_{i}\right), z, y \in \operatorname{cl} B\left(0, \max \left\{n r_{i-1}^{* *}(t), n r_{i}^{*}(t)\right\}\right)$, and for $t \in$ $\left(\sigma_{i}+b_{i}, \sigma_{i+1}\right), z, y \in \operatorname{cl} B\left(0, r_{i}^{* *}(t)\right),\left(\left(H^{m}\right)^{2} \leq 8 n\left(\max \left\{r_{i-1}^{\prime \prime}, r_{i}^{\prime \prime}\right\}\right)\right.$ when $t \in\left(\sigma_{i}+M_{i}, \sigma_{i}+b_{i}\right], t<T+M, z, y \in$ $\left.\operatorname{cl} B\left(0, \max \left\{n r_{i-1}^{* *}(t), n r_{i}^{* *}(t)\right\}\right)\right)$. Finally, in this nonjumping system, the criterion is $E \int_{0}^{T+M} h_{0}(t, z, v, \sigma) \mathrm{d} t$. Theorem 2.1, with Remark $2.4\left(A=\left\{(x, x): x \in \mathbb{R}^{n}\right\}\right)$, implies the existence of an optimal control $u_{*}(t, \sigma)$ in this system, which implies the existence of an optimal control $u^{*}(t, \tau)$ in the original jumping system.

B. Consider next the case where $|g| \leq M_{i}, \sum \sqrt{M_{i}}<\infty$ is not satisfied. For any $i$, there exist positive nondecreasing continuous functions $r_{i}($.$) and positive numbers K_{i}$ and $\bar{k} \in\left(1,1 / k_{*}\right)$ such that $\left|f_{0}(t, x, u, \tau)\right|,|f(t, x, u, \tau)|$ $\leq K_{i}$ when $x \in \operatorname{cl} B\left(0, r_{i}(t)\right),(u, \tau) \in U \times \Omega^{\prime \prime}, t \in\left(\tau_{i}, \tau_{i+1}\right)$, with $\sup _{i} K_{i} / \bar{k}^{i}<\infty, \sup _{i, t \in[0, T]} r_{i}(t) / \bar{k}^{i}<\infty$. Moreover, the following property holds: for any $u(.,.) \in U^{\prime}$, for any $\tau \in \Omega^{\prime \prime}$, for any $\bar{x} \in \operatorname{clB}\left(0, r_{i-1}\left(\tau_{i}\right)\right)$, any solution $x^{u}\left(t, \tau ; \tau_{i}, \bar{x}\right)$ of $(2.2),(3.1)$ on $\left[\tau_{i}, T\right]$ with $x^{u}\left(\tau_{i}-, \tau ; \tau_{i}, \bar{x}\right)=\bar{x}$, satisfies $\left|x^{u}\left(t, \tau ; \tau_{i}, \bar{x}\right)\right| \leq r_{j}(t)$ when $t \in\left(\tau_{j}, \tau_{j+1}\right)$. Also $\left|x^{u}(t, \tau ; 0,0)\right| \leq r_{j}(t)$ when $t \in\left(\tau_{j}, \tau_{j+1}\right)$, for any solution $x(y, \tau ; 0,0)$ on $[0, T]$.

To see this, choose numbers $\kappa^{\prime}>\kappa$ and $\kappa_{g}^{\prime}>\kappa_{g}, \kappa_{g}^{\prime} \geq 1$, such that $k_{*}<1 / \kappa_{g}^{\prime}$, and let $\beta:=\max \left\{\alpha_{g} /\left(\kappa_{g}^{\prime}-\right.\right.$ $\left.\left.\kappa_{g}\right), \alpha /\left(\kappa^{\prime}-\kappa\right)\right\}$. When $|x| \geq \beta$, then $\kappa_{g}^{\prime}|x| \geq \alpha_{g}+\kappa_{g}|\tilde{x}|$ and $\kappa^{\prime}|x| \geq \alpha+\kappa|x|$. For any $u(.,$.$) , when |\tilde{x}| \leq$ $\beta\left(\kappa_{g}^{\prime}\right)^{k-1} \mathrm{e}^{\kappa^{\prime} \tau_{k}}$, then

$$
\left|x^{u}\left(\tau_{k}+, \tau ; \tau_{k}, \tilde{x}\right)\right| \leq \alpha_{g}+\kappa_{g} \beta\left(\kappa_{g}^{\prime}\right)^{k-1} \mathrm{e}^{\kappa^{\prime} \tau_{k}} \leq \beta\left(\kappa_{g}^{\prime}\right)^{k} \mathrm{e}^{\kappa^{\prime} \tau_{k}},
$$

and for $t \in\left(\tau_{k}, \tau_{k+1}\right]$, by Gronwall's inequality, $\left|x^{u}\left(t, \tau ; \tau_{k}, \tilde{x}\right)\right| \leq \beta\left(\kappa_{q}^{\prime}\right)^{k} \mathrm{e}^{\kappa^{\prime} \tau_{k}} \mathrm{e}^{\kappa^{\prime}\left(t-\tau_{k}\right)}=\beta\left(\kappa_{q}^{\prime}\right)^{k} \mathrm{e}^{\kappa^{\prime} t}$, where $x^{u}\left(t, \tau ; \tau_{k}, \tilde{x}\right)$ is any solution starting at $\left(\tau_{k}-, \tilde{x}\right)$ corresponding to $u(.,$.$) . Moreover,$

$$
\begin{aligned}
\left|x^{u}\left(\tau_{k+1}+, \tau ; \tau_{k}, \tilde{x}\right)\right| & \leq\left|\hat{g}\left(\tau_{k+1}, x^{u}\left(\tau_{k+1}-, \tau ; \tau_{k}, \tilde{x}\right), k+1\right)\right| \\
& \leq \alpha_{g}+\kappa_{g} \beta\left(\kappa_{g}^{\prime}\right)^{k} \mathrm{e}^{\kappa^{\prime} \tau_{k+1}} \\
& \leq \kappa_{g}^{\prime} \beta\left(\kappa_{g}^{\prime}\right)^{k} \mathrm{e}^{\kappa^{\prime} \tau_{k+1}}=\beta\left(\kappa_{g}^{\prime}\right)^{k+1} \mathrm{e}^{\kappa^{\prime} \tau_{k+1}},
\end{aligned}
$$

so for $t \in\left(\tau_{k+1}, \tau_{k+2}\right)$,

$$
\left|x^{u}\left(t, \tau, ; \tau_{k}, \tilde{x}\right)\right| \leq\left[\beta\left(\kappa_{g}^{\prime}\right)^{k+1} \mathrm{e}^{\kappa^{\prime} \tau_{k+1}}\right] \mathrm{e}^{\kappa^{\prime}\left(t-\tau_{k+1}\right)}=\beta\left(\kappa_{g}^{\prime}\right)^{k+1} \mathrm{e}^{\kappa^{\prime} t} .
$$


Continuing in this manner, it is easily seen that for $i>k$, when $t \in\left(\tau_{i}, \tau_{i+1}\right)$ and $|\tilde{x}| \leq \beta\left(\kappa_{g}^{\prime}\right)^{k-1} \mathrm{e}^{\kappa^{\prime} \tau_{k}}$, then

$$
\left|x^{u}\left(t, \tau ; \tau_{k}, \tilde{x}\right)\right| \leq \beta\left(\kappa_{g}^{\prime}\right)^{k} \mathrm{e}^{\kappa^{\prime} \tau_{k}}\left(\kappa_{g}^{\prime}\right)^{i-k} \mathrm{e}^{\kappa^{\prime}\left(t-\tau_{k}\right)} \leq \beta \mathrm{e}^{\kappa^{\prime} t}\left(\kappa_{g}^{\prime}\right)^{i}=: r_{i}(t)
$$

Finally, put $K_{i}=\alpha^{\prime}+\kappa \sup _{t \in[0, T]} r_{i}(t)$. The existence of functions $r_{i}(t)$ and numbers $K_{i}$ with the above properties has then been shown, (for any $\left.\bar{k} \in\left(\kappa_{g}^{\prime}, 1 / k_{*}\right)\right)$.

Note also that $\beta \geq \alpha_{g} /\left(\kappa_{g}^{\prime}-\kappa_{g}\right)$, hence $|\hat{g}(t, x, i)| \leq \alpha_{g}+\kappa_{g}|x| \leq \kappa_{g}^{\prime} \beta^{\prime}$ when $|x| \leq \beta^{\prime}, \beta^{\prime} \geq \beta$, so

$$
|\hat{g}(t, x, i)| \leq \kappa_{g}^{\prime} n r_{i^{\prime}}(t) \text {, when }|x| \leq n r_{i^{\prime}}(t) .
$$

Choose a decreasing sequence $d_{i} \in(0,1]$, with $d_{0}=1$, such that $M_{i}:=\max \left\{d_{i-1}\left(\kappa_{g}^{\prime}+\right.\right.$ 1) $\left.n \sup _{t \in[0, T]} r_{i-1}(t), d_{i}\left(\kappa_{g}^{\prime}+1\right) n \sup _{t \in[0, T]} r_{i}(t)\right\}$ satisfies $\sum_{i=1}^{\infty} \sqrt{M_{i}}<\infty$. Consider now the system

$$
\dot{y}=\hat{f}(t, y, u, \tau):=\sum_{i=0}^{\infty} d_{i} f\left(t, y / d_{i}, u, \tau\right) 1_{\left[\tau^{i}, \tau^{i+1}\right)}(t), y(0)=0,
$$

$\hat{f}_{0}(t, y, u, \tau):=\sum_{i=0}^{\infty} f_{0}\left(t, y / d_{i}, u, \tau\right) 1_{\left[\tau^{i}, \tau^{i+1}\right)}(t)$, with jumps governed by $y\left(\tau_{i}+\right)=\check{g}\left(\tau_{i}, y\left(\tau_{i}-\right), i\right):=$ $d_{i} \hat{g}\left(\tau_{i}, y\left(\tau_{i}-\right) / d_{i-1}, i\right)$ and with end conditions $y^{m}(T)=0, m=1, \ldots, n_{1}, y^{m}(T) \geq 0, m=n_{1}+1, \ldots, n_{2}$. Evidently, $\left|\hat{f}_{0} 1_{\left[\tau^{i}, \tau^{i+1}\right)}(t)\right|=\left|f_{0}\left(t, y / d_{i}, u, \tau\right)\right| 1_{\left[\tau^{i}, \tau^{i+1}\right)}(t) \leq K_{i}$ when $|y| \leq d_{i} r_{i}(t)$, (then $\left|y / d_{i}\right| \leq r_{i}(t)$ ) Moreover, $\left|\hat{f} 1_{\left[\tau^{i}, \tau^{i+1}\right)}(t)\right|=\left|d_{i} f\left(t, y / d_{i}, u, \tau\right)\right| 1_{\left[\tau^{i}, \tau^{i+1}\right)}(t) \leq d_{i} K_{i} \leq K_{i}$, when $|y| \leq d_{i} r_{i}(t)$. Now, for any solution $x^{u}\left(t, \tau ; \tau_{k}, \tilde{x}\right)$, the function $y^{u}\left(t, \tau ; \tau_{k}, \tilde{y}\right)$, defined by $y^{u}\left(t, \tau ; \tau_{k}, \tilde{y}\right)=d_{i} x^{u}\left(t, \tau ; \tau_{k}, \tilde{y}\right)$ for $t \in\left(\tau_{i}, \tau_{i+1}\right], \tilde{y}=d_{k-1} \tilde{x}$, is a solution of this new system (in particular $y^{u}\left(\tau_{i}+, \tau ; \tau_{k}, \tilde{y}\right)=d_{i} x^{u}\left(\tau_{i}+, \tau ; \tau_{k}, \tilde{y}\right)=d_{i} \hat{g}\left(\tau_{i}, x^{u}\left(\tau_{i}-, \tau ; \tau_{k}, \tilde{y}\right), i\right)=$ $\left.d_{i} \hat{g}\left(\tau_{i}, y^{u}\left(\tau_{i}-, \tau ; \tau_{k}, \tilde{y}\right) / d_{i-1}, i\right)=\check{g}\left(\tau_{i}, y^{u}\left(\tau_{i}-, \tau ; \tau_{k}, \tilde{y}\right), i\right), i \geq k\right)$. Then, evidently, $\left|y^{u}\left(t, \tau ; \tau_{k}, \tilde{y}\right)\right| \leq d_{j} r_{j}(t)$ for $t \in\left(\tau_{j}, \tau_{j+1}\right]$ when $|\tilde{y}| \leq d_{k-1} r_{k-1}(t)$. Finally, by (3.5), when $|y| \leq d_{i-1} n r_{i-1}(t)$, then

$$
|\check{g}(t, y, i)-y|=\left|d_{i} \hat{g}\left(t, y / d_{i-1}, i\right)-y\right| \leq d_{i} \kappa_{g}^{\prime} n r_{i-1}(t)+d_{i-1} n r_{i-1}(t) \leq d_{i-1} n r_{i-1}(t)\left(\kappa_{g}^{\prime}+1\right) \leq M_{i},
$$

and for $|y| \leq d_{i} n r_{i}(t)$,

$$
|\check{g}(t, y, i)-y|=\left|d_{i} \hat{g}\left(t, y / d_{i-1}, i\right)-y\right| \leq d_{i} \kappa_{g}^{\prime} n r_{i}(t)+d_{i} n r_{i}(t) \leq d_{i} n r_{i}(t)\left(\kappa_{g}^{\prime}+1\right) \leq M_{i} .
$$

For $r_{i}^{*}(t)=d_{i} r_{i}(t)$, the system $\left(\hat{f}_{0}, \hat{f}, \check{g}\right)$ satisfies the auxiliary conditions in A, so an optimal pair $\left(y^{*}(.,),. u^{*}(.,).\right)$ exists. Defining $x^{*}(t)=y^{*}(t) / d_{i}$ for $t \in\left(\tau_{i}, \tau_{i+1}\right]$, then $\left(x^{*}(.,),. u^{*}(.,).\right)$ is optimal in the original jumping system, ((2.3) and (2.4) are satisfied because $y^{*}(t, \tau)$ satisfies $y^{* i}(t, \tau)=0, i=1, \ldots, n_{1}$ and $y^{* i}(t, \tau) \geq 0, i=$ $n_{1}+1, \ldots, n_{2}$ a.s. Thus $\sum_{i} x^{*}(T, \tau) d_{i} 1_{\left[\tau_{i}, \tau_{i+1}\right)}(T)$, and so also $x^{*}(T, \tau) d_{i} 1_{\left[\tau_{i}, \tau_{i+1}\right)}(T)$ and hence $x^{*}(T, \tau)$, satisfy the same relationships a.s.).

Remark 3.3. A question has been raised if, in my setting, a proof would be more rapidly constructed if one made use of infinite horizon, autonomous discrete time dynamic programming results, as Davis [3] does. In Davis [3], Bertsekas and Shreve [1] is used and there the criterion is required to be nonpositive when a maximization is carried out. One would need to rewrite the problem to be Markovian, using a state $y$ to represent time, and a jumping state $z$ with an infinite number of coordinate to represent $\left(\tau_{1}, \tau_{2}, \ldots\right.$ ) (at jump $k$ it equals $\left(\tau_{1}, \ldots, \tau_{k}, 0,0, \ldots\right)$. As I don't want to use nonpositivity of the criterion, the last mentioned book could be replaced by Hernandez-Lerma et al. [9], as my problem yields a transient discrete time model in the sense of that paper. However, if one aims at Borel measurable controls and the dynamics are given by difference equations, usc, and not only measurability, of the optimal value function is needed. If, for some suitable metric on the set of states $z$, the optimal value function as a function of $(x, z)$ is going to be usc, then in particular we need to know that $\left(x, \tau^{k}\right) \rightarrow V^{k, \infty}\left(x, \tau^{k}\right)$ is usc for any $k$, i.e. we would surely need the proof presented above for this fact. To go between the discrete time and the continuous time model, compactness and measurable selection results as exemplified by Proposition 2.2 and the arguments subsequent to (2.29) would again be needed. Even if possible, it seems that not much would be gained by using such an approach. 
Acknowledgements. I am very grateful to two referees whose comments made it possible for me to improve the exposition and remove errors.

\section{REFERENCES}

[1] D. Bertsekas and S.E. Shreve, Stochastic optimal control: the discrete-time case. Academic Press, New York (1978).

[2] L. Cesari, Optimization - Theory and Applications. Springer-Verlag, New York (1983).

[3] M.H.A. Davis, Markov Models and Optimization. Chapman \& Hall, London, England (1993).

[4] M.H.A. Davis and M. Farid, Piecewise deterministic processes and viscosity solutions, in Stochastic analysis, control, optimization and applications, edited by W.M. Mc.Eneaney et al., A volume in honour of W.H. Fleming, on occation of his 70th birthday, Birkhäuser, Boston (1999) 249-286.

[5] M.A.H. Dempster, Optimal control of piecewise-deterministic Markov Processes, in Applied Stochastic Analysis, edited by M.A.H. Davis and R.J. Elliot. Gordon and Breach, New York (1991) 303-325.

[6] M.A.H. Dempster and J.J. Ye, A maximum principle for control of piecewise deterministic Markov Processes, in Approximation, optimization and computing: Theory and applications, edited by A.G. Law et al., NorthHolland, Amsterdam (1990) 235-240.

[7] M.A.H. Dempster and J.J. Ye, Necessary and sufficient optimality conditions for control of piecewise deterministic Markov processes. Stoch. Stoch. Rep. 40 (1992) 125-145.

[8] L. Forwick, M. Schäl and M.Schmitz, Piecewise deterministic Markov control processes with feedback controls and unbounded costs, Acta Appl. Math. 82 (2004) 239-267.

[9] O. Hernandez-Lerma et al., Markov processes with expected total cost criterion: optimality, stability, and transient models. Acta Appl. Math. 59 (1999) 229-269.

[10] A. Seierstad, Stochastic control in discrete and continuous time. Springer, New York, NY (2009).

[11] A. Seierstad, A stochastic maximum principle with hard end constraints. J. Optim. Theory Appl. 144 (2010) 335-365.

[12] D. Vermes, Optimal control of piecewise-deterministic Markov processes. Stochastics 14 (1985) 165-208.

[13] J.J. Ye, Generalized Bellman-Hamilton-Jacobi equations for piecewise deterministic Markov Processes, , in Systems modelling and optimization, Proceedings of the 16th IFIP-TC conference, Compiegne, France, July 5-9 1993, Lect. Notes Control Inf. Sci. 197, edited by J. Henry et al., London, Springer-Verlag (1994) 51-550.

[14] J.J. Ye, Dynamic programming and the maximum principle of piecewise deterministic Markov processses, in Mathematics of stochastic manufacturing systems, AMS-SIAM summer seminar in applied mathematics, June 17-22 1996, Williamsburg, VA, USA, Lect. Appl. Math. 33, edited by G.G. Yin et al., Amer. Math. Soc., Providence, RI (1997) 365-383. 